\title{
A Method of the Riemann-Hilbert Problem for Zhang's Conjecture 2 in a Ferromagnetic 3D Ising Model: Topological Phases
}

\author{
Zhidong Zhang ${ }^{1, *(\mathbb{D})}$ and Osamu Suzuki ${ }^{2}$ \\ 1 Shenyang National Laboratory for Materials Science, Institute of Metal Research, Chinese Academy of Sciences, \\ 72 Wenhua Road, Shenyang 110016, China \\ 2 Department of Computer and System Analysis, College of Humanities and Sciences, Nihon University, \\ Sakurajosui 3-25-40, Setagaya-ku, Tokyo 156-8550, Japan; osuzuki1944butterfly@gmail.com \\ * Correspondence: zdzhang@imr.ac.cn; Tel.: +86-24-23971859
}

Citation: Zhang, Z.; Suzuki, O. A Method of the Riemann-Hilbert Problem for Zhang's Conjecture 2 in a Ferromagnetic 3D Ising Model: Topological Phases. Mathematics 2021, 9, 2936. https://doi.org/10.3390/ math9222936

Academic Editor: Jan Sładkowski

Received: 3 September 2021

Accepted: 2 November 2021

Published: 18 November 2021

Publisher's Note: MDPI stays neutral with regard to jurisdictional claims in published maps and institutional affiliations.

Copyright: (C) 2021 by the authors. Licensee MDPI, Basel, Switzerland. This article is an open access article distributed under the terms and conditions of the Creative Commons Attribution (CC BY) license (https:// creativecommons.org/licenses/by/ $4.0 /)$.

\begin{abstract}
A method of the Riemann-Hilbert problem is employed for Zhang's conjecture 2 proposed in Philo. Mag. 87 (2007) 5309 for a ferromagnetic three-dimensional (3D) Ising model in a zero external magnetic field. In this work, we first prove that the 3D Ising model in the zero external magnetic field can be mapped to either a $(3+1)$-dimensional $((3+1) \mathrm{D})$ Ising spin lattice or a trivialized topological structure in the $(3+1) \mathrm{D}$ or four-dimensional (4D) space (Theorem 1). Following the procedures of realizing the representation of knots on the Riemann surface and formulating the RiemannHilbert problem in our preceding paper [O. Suzuki and Z.D. Zhang, Mathematics 9 (2021) 776], we introduce vertex operators of knot types and a flat vector bundle for the ferromagnetic 3D Ising model (Theorems 2 and 3). By applying the monoidal transforms to trivialize the knots/links in a 4D Riemann manifold and obtain new trivial knots, we proceed to renormalize the ferromagnetic 3D Ising model in the zero external magnetic field by use of the derivation of Gauss-Bonnet-Chern formula (Theorem 4). The ferromagnetic 3D Ising model with nontrivial topological structures can be realized as a trivial model on a nontrivial topological manifold. The topological phases generalized on wavevectors are determined by the Gauss-Bonnet-Chern formula, in consideration of the mathematical structure of the 3D Ising model. Hence we prove the Zhang's conjecture 2 (main theorem). Finally, we utilize the ferromagnetic 3D Ising model as a platform for describing a sensible interplay between the physical properties of many-body interacting systems, algebra, topology, and geometry.
\end{abstract}

Keywords: ferromagnetic 3D Ising model; topological phase; Gauss-Bonnet-Chern formula; RiemannHilbert problem; vertex operators; vector bundle

\section{Introduction}

The Ising model has attracted intensive interest since the 1920s [1], which not only applies to the interpretation of phase transitions and critical phenomena in different fields, but also provides a fundamental understanding on interactions and dimensionality. The Ising model can be utilized to describe many-body interacting spin (particle) systems in condensed matter physics, statistical physics, high energy physics, particles physics, mathematical physics, etc. Furthermore, the Ising model can be mapped to modelling the behaviors of systems in biology, computer sciences, economics, and sociology. A great progress in this field is that Onsager derived the exact solution of a two-dimensional (2D) Ising model in the zero external magnetic field, in which no nontrivial topological structures exist [2]. The exact solution of a three-dimensional (3D) Ising model in the zero external magnetic field had been a well-known 100-year problem in physics. The first author (ZDZ) made an observation that nontrivial topological structures exist in the formula of the partition functions of the 3D Ising lattices in the zero external magnetic field for any positive inverse temperature [3,4]. Zhang conjectured that the nontrivial knot/link structures of the ferromagnetic 3D Ising model in the zero external magnetic 
field can be trivialized in higher dimensional space and it can be realized as the free statistic model on the $(3+1)$-dimensional (i.e., $(3+1) \mathrm{D})$ space with topological/geometrical phases on eigenvectors $[3,4]$. Ławrynowicz and coworkers reformulated the algebraic part of the quaternion approach used in [3] in terms of the quaternionic sequence of Jordan algebras, in order to investigate the geometrical aspects, related fractals, and chaos of simple orthorhombic Ising lattices [5,6]. Zhang, Suzuki and March developed a Clifford algebraic procedure for the ferromagnetic 3D Ising model in the zero external magnetic field [7], in which we proved four theorems (Trace Invariance Theorem, Linearization Theorem, Local Transformation Theorem, Commutation Theorem). The Zhang-Suzuki-March approach [7] has rigorously proven the Zhang's two conjectures already, verifying the correctness of Zhang's exact solution. In a recent work [8], the lower bound of the computational complexity of the spin-glass 3D Ising system was determined, which was based on a deep understanding of the mathematical structure of the Ising models on 3D lattices. More recently, the exact solution of the transverse-field ferromagnetic/antiferromagnetic 2D Ising model was derived by equivalence between this transverse-field 2D Ising model and the ferromagnetic/antiferromagnetic 3D Ising model [9].

In the preceding paper [10] of the present series, we developed a method of the Riemann-Hilbert problem for Zhang's conjecture 1 proposed in [3] and rigorously proved (by a new approach) Zhang's conjecture 1 in the following steps [10]: (1) the Clifford algebra $\mathrm{Cl}\left(\mathrm{I}_{3 \mathrm{D}}\right)$ is extended to the Knot/Clifford $(\mathrm{K} / \mathrm{C})$ algebra which has the original Clifford algebra and its conjugate algebra $\overline{C l\left(I_{3 D}\right)}$ as subalgebras [10]; and (2) The K/C knot $Z_{\gamma}$ is extended to the $K / C$ algebra, which is denoted by $\sigma\left(Z_{\gamma}, \bar{Z}_{\gamma}\right)$. Hence, a knot carries the elements in $\mathrm{K} / \mathrm{C}$ algebra for the partition function of the ferromagnetic $3 \mathrm{D}$ Ising model [10-20]. (3) We realize the knots/links of the 3D Ising model on Riemann surfaces as a monodromy representation [10]. (4) We formulate the Riemann-Hilbert problem on the Riemann surfaces for the representation and obtain analytic solutions with regular singularities at the knot/link intersection points $[10,21,22]$. (5) Applying the monoidal transformation $[10,23,24]$, which is developed by the resolution of singularities to the functions in (4), we construct the desired trivialization in $\mathrm{K} / \mathrm{C}$ algebra, which solves Zhang's conjecture 1. Using these processes, we prove the following theorem [10]:

Suzuki-Zhang Theorem [10]: The Zhang's conjecture 1 proposed in [3] can be solved. Namely, for a given K/C knot which is given by the partition function of the ferromagnetic $3 D$ Ising model in the zero external magnetic field, we can make the trivialization by the use of monoidal transform trivialization.

In this paper, we continue our work on the trivialization of nontrivial topological structures of the 3D Ising model in the zero external magnetic field; however, we are concerned mainly with Zhang's conjecture 2 proposed in [3]. At first, in Section 2, we investigate the $3 \mathrm{D}$ Ising model by a mapping between a knot and a spin lattice. The mapping provides a concise proof of Zhang's conjectures 1 and 2. This procedure is helpful for understanding the contribution of nontrivial topological structures to the partition function and physical properties of the ferromagnetic 3D Ising model, and the spontaneous emergence of the additional dimension, and the connection between different approaches developed based on topology and algebra. Then, we focus our interest on a detailed proof of Zhang's conjecture 2 by formulating the Riemann-Hilbert problem and by using the GaussBonnet-Chern formula. In Section 3, we describe the representation of the fundamental group. Following the processes developed in [10], i.e., realizing the representation on the Riemann surface and formulating the Riemann-Hilbert problem, we introduce vertex operators of knot types and a flat vector bundle of the 3D Ising model in Sections 4 and 5 respectively. For constructions of the trivialization, as indicated in [10], we apply the method of monoidal transforms to trivialize the knots/links in a 4D space and obtain new trivial knots. In Section 6, we proceed to obtain the renormalized model of the 3D Ising model. In order to realize models without singularities, we have to take off nontrivial knots in a higher dimensional space. This can be performed by use of the derivation of the Gauss-Bonnet-Chern formula. We make the renormalization of the model and have the 
curvature structure in the model. The ferromagnetic 3D Ising model in the zero external magnetic field with nontrivial topological structures can be realized as the trivial model on a nontrivial topological manifold, while three topological phases $\phi_{x}=2 \pi, \phi_{y}=\phi_{z}=\pi / 2$ appear on the quaternionic eigenvectors of the 3D Ising model. Hence we rigorously prove Zhang's conjecture 2 (main theorem). In Section 7 , we summarize our results and utilize the 3D Ising model to investigate the interplay between the physical properties of many-body interacting spin systems, algebra, topology, and geometry. In Section 8, the conclusion is given. The details for the proofs of Theorems 2 and 4 are represented in Appendices A and B, respectively.

\section{Mapping between Knots and Spin Lattices in the 3D Ising Model}

In this section, we first recall the facts of topology that a knot can be mapped to a spin lattice, and the crossings of the knot can contribute to the physical properties of the spin lattice, the same as what spins do. Then, we apply this mapping to the ferromagnetic 3D Ising model, and show that there are two kinds of contributions (local environments of spin alignments and nonlocal global effects of knots) to the partition function of the 3D Ising system. Finally, we view the 3D Ising model from two angles of the spin alignments and the topological structures, apply the two transformations mapping from $3 \mathrm{D}$ to $(3+1) \mathrm{D}$, and prove the equivalence of the two mappings (see Theorem 1).

\subsection{Mapping between a Knot and a Spin Lattice}

Let us consider a knot that is embedded in a 3D space (imagine, e.g., a piece of rope). Following the standard treatment [13-20], one can project the knot onto the plane and obtain a 2D-knot diagram. The essential topological information about the knot is embodied in the pattern of crossings. Clearly, a crossing takes values $b_{k}= \pm 1$ [13-20], which corresponds to a fact that in the 2D image, one rope segment goes over or under another rope segment. Figure 1 shows that a crossing can be mapped to one of two spin states (up and down) with values +1 and -1 . It is well known that the crossing state (there are two possible states for a crossing $(\times)$ ) of a knot can be mapped to the spin state (there are two possible states for an Ising spin) of a lattice, while the crossing point is mapped to a lattice point [13-20]. There is a one-to-one mapping between a knot and a spin lattice [13-20].

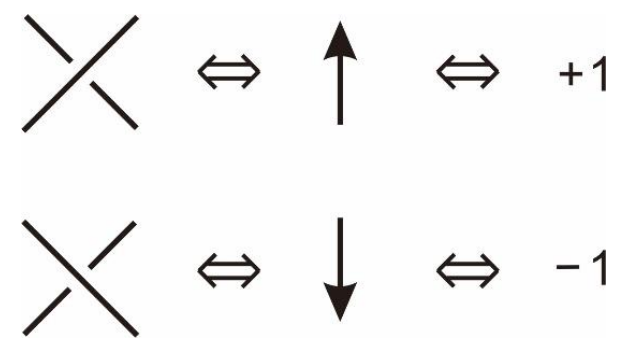

Figure 1. Mapping between a state of crossings and a state of Ising spins. Two crossing states are mapped to two spin states (up and down) with values of +1 and -1 , respectively.

One can make a connection of the crossings of knots to spin glasses by assigning quenched random values to the crossing variables $b_{k}$, leading to the links crossing above and below at random. The Kauffman bracket polynomial is identical to the partition function of a Potts model (including an Ising model), but up to an irrelevant multiplicative factor. It was illustrated that the $2 \mathrm{D}$-knot diagram lives on a lattice $M$ consisting of lines oriented at $\pm 45^{\circ}$, intersecting at the crossings $b_{k}$, that carry the disorder. A dual lattice $\mathrm{L}$, rotated by $45^{\circ}$, can be defined. Its horizontal and vertical edges (denoted $b_{i j}$ ) are in one-toone correspondence with the vertices $b_{k}$ of the lattice $M$ [20]. Figure 2 illustrates an example of the 2D-knot diagram with randomly distributed crossings on a lattice $M$, which can be mapped to spins located on a dual lattice $L$ with randomly distributed spin states. The Ising spin states $s_{i}$ are associated with knot properties in an abstract manner. Certainly, the 
duality between the knot structure and the spin lattice can be generalized to the $3 \mathrm{D}$ case. The Kauffman bracket polynomial variable $A$ can be used to describe their characterizations, which is a weight for the manner in which a set of microstates are assembled into the 2Dknot diagram, and vice versa (namely, the 2D-knot diagram is disassembled into a set of microstates). Moreover, they are also correlated with the Jones' polynomial variable $t$ [17]. Note that the Kauffman and Jones bracket polynomials coincide when $A^{1 / 4}=t[13-20]$.
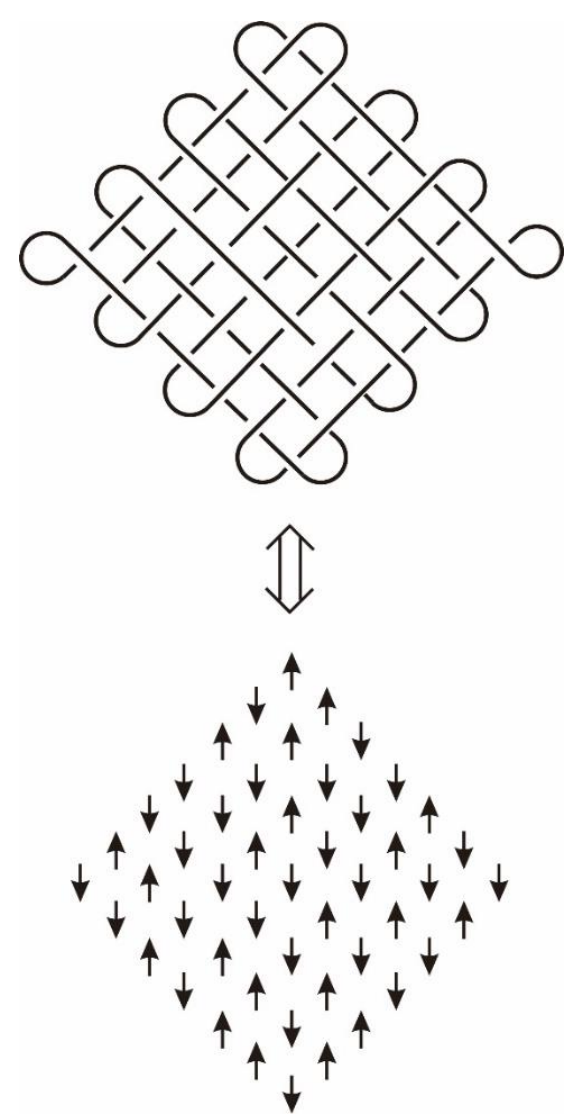

Figure 2. Mapping between the 2D-knot diagram with randomly distributed crossings on a lattice $M[20]$ and a spin dual lattice $L$.

If the crossing states of the knot are randomly distributed, it will correspond to a spin-glass Ising model [8] or a paramagnetic state in the ferromagnetic model. Note that the knot state is equivalent to the spin-glass state, and even frustration may present in the spin-glass state (see Figure 2). This is because the mapping between a knot and a spin glass is a one-to-one mapping. The frustration presenting in the spin-glass system is just the result of competition between randomly distributed inter-spin coupling constants, while in knots the frustration may also be present if one considers the competitive couplings between crossings. If the crossing states of the knot are in a certain order manner, it may correspond to a ferromagnetic state in the Ising model.

According to the topology theory [13-20], a state of the knot diagram is very similar to the energetic states of a physical system. As the system is deformed topologically, one can preserve the state structure, making invariant properties of states become topological invariants of the knot or link. For a given system, the topological evolution of states and the integration over the space of states are complementary for investigating the topology of knots and links. There are two choices for topologically smoothing a given crossing $(\times)$, and thus $2^{N}$ states of a diagram exist with $N$ crossings [13-20] (same as $2^{N}$ states of a spin lattice with $N$ spins). The bracket polynomial (namely, the state summation) for knots and links is defined as [13-15]:

$$
\langle K\rangle=\sum_{\sigma}\langle K \mid \sigma\rangle d^{|\sigma| \mid}
$$


which is an analog of a partition function in discrete statistical mechanics. $\sigma$ runs over all the states of $K . d=-A^{2}-B^{2}$, with $A, B$, and $d$ being commuting algebraic variables. The bracket state summation can be utilized to express the partition function for the Potts model (with $q$ spin states for each Potts spin) for appropriate choices of commuting algebraic variables [13-20]. This is also true for the Ising model, since the Ising model is a Potts model with $q=2$. As revealed in [4], the nontrivial topological basis can be transformed to the trivial topological basis by a transformation (a matrix or a rotation), and vice versa:

$$
\left[\begin{array}{c}
\langle\chi\rangle \\
\left\langle\chi^{-1}\right\rangle
\end{array}\right]=\left[\begin{array}{cc}
A & B \\
B & A
\end{array}\right]\left[\begin{array}{c}
\left\langle\begin{array}{c}
\cup \\
\cap
\end{array}\right\rangle \\
\langle()(\rangle
\end{array}\right]
$$

with its reverse

$$
\left[\begin{array}{c}
\left\langle\begin{array}{c}
\cup \\
\cap
\end{array}\right\rangle \\
\langle(\rangle)
\end{array}\right]=\frac{1}{A^{2}-B^{2}}\left[\begin{array}{cc}
A & -B \\
-B & A
\end{array}\right]\left[\begin{array}{c}
\langle\chi\rangle \\
\left\langle\chi^{-1}\right\rangle
\end{array}\right]
$$

It is well known that under the Reidemeister moves II and III, the bracket with $B=A^{-1}, d=-A^{2}-A^{-2}$ is invariant. As long as nontrivial knots or links exist in a system, as revealed in [4], a matrix representing such a transformation (i.e., a rotation) intrinsically and spontaneously may always exist, no matter how complicated the knots or links are. This topology fact is employed in the recent work [7] to prove the Local Transformation Theorem, in order to trivialize the nontrivial topological structure in the 3D Ising model.

Remark 1. It is hard to derive the explicit expression of the topological contribution to the partition function and the thermodynamic properties by the above transformation from the nontrivial topological basis to the trivial topological basis, because the nontrivial topological structures are very complicated and it is difficulty to account every crossing term by term. One may refer to [3,7] for the results of these physical properties obtained by the local transformation.

\subsection{Mapping between Knots and Spin Lattices in the 3D Ising Model}

From the facts above, we have already understood that the topological structure can contribute to the partition function and physical properties of a physical system. In the following, we shall show that besides the contribution of spin alignments, there is an additional contribution, due to the nontrivial topological structure in the 3D Ising systems. In the ferromagnetic 3D Ising model we are studying in this work, a nontrivial topological structure in the representation of $\Gamma$-matrices exists (with Clifford algebra), which is caused by the so-called internal factors (i.e., the nonlinear terms) in the transfer matrices $[3,4,7,10,12]$. The trivial topological states are defined as a circle (or an interval) and a knot of type I that was illustrated in Figure 2 of our preceding paper [10], which correspond to the linear terms in the transfer matrices $\mathbf{V}_{\mathbf{1}}$ and $\mathbf{V}_{\mathbf{2}}$ of the ferromagnetic 3D Ising model. The nontrivial topological states are defined as a braid with many crossings, which is called the basic form of knot type II, as shown in Figure 3 of [10] (see also the braid in Figure 3 of this paper). Combined with a knot $\gamma$ constructed in the 3D lattice $\mathrm{Z}_{3}$ (see Figure 1 of [10]), we have the nontrivial topological states illustrated schematically in Figure 4 of [10].

There are two kinds of contributions to the partition function of the 3D Ising system: (1) local environments of spin alignments and (2) nonlocal global effects of knots caused by many-body interactions and dimensionality. Only is the contribution of the local environments of spin alignments clearly visible in the spin representation; however, the two contributions are clearly visible in the $\Gamma$-matrix representation. In the $\Gamma$-matrix representation, the transfer matrix $V_{3}$ shows that besides the local interaction that is the same as that in $\mathbf{V}_{\mathbf{1}}$ and $\mathbf{V}_{\mathbf{2}}$, a long-range effective interaction (entanglement) between spins in a plane also exists, namely, the so-called nontrivial topological structure, and it is represented by a braid $[7,10]$. Since the formulas in the two representations (i.e., spin representation and $\Gamma$-matrix representation) are connected by a series of equalities [7], both 
the contributions exist simultaneously in the system, no matter which representation we use. Such a long-range spin entanglement is caused by the planar character of the matrix used for the transfer matrices in quantum statistical mechanics and the arrangement of spins in a 3D lattice. Figure 3 illustrates a unit cell of a cubic Ising lattice, in which a spin is located at every lattice site and a braid connects a pair of the two nearest neighboring sites along the third dimension. The schematic representation in Figure 3 is equivalent to that illustrated in Figure 4 of [10].

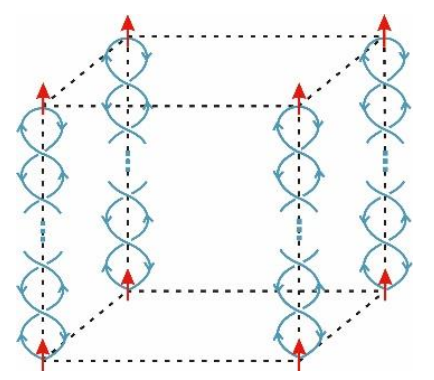

Figure 3. A unit cell of the 3D cubic Ising lattice (black dashed lines) with spins (red arrows) located at every lattice site and braids (blue curves) attached to every pair of the two nearest neighboring lattice sites along the third dimension. The spins represent the linear terms in the transfer matrices, while the braids represent the nontrivial knots, namely, the nonlinear terms of $\Gamma$-matrices of the transfer matrices, of the 3D Ising lattice.

\subsection{Viewing the 3D Ising Model from an Angle of the Spin Alignments}

On the one hand, one can view the 3D Ising model from an angle of the spin alignments. According to the topology theory [13-20], the nontrivial topological structure (i.e., nonlocal effects) in the representation of $\Gamma$-matrices can be mapped to a spin Ising lattice. Figure 4 shows that a braid, the basic element of the nontrivial topological structure, can be mapped to a spin-chain lattice. This means that besides the original 3D Ising lattice (with spin alignments), an additional "imagine" Ising spin lattice also exists, which forms because of the mapping from nontrivial knots of $\Gamma$-matrices of the transfer matrices. The original 3D Ising lattice and the "imagine" Ising lattice together construct a composited "big" Ising spin lattice (see Figure 5 for a cubic lattice as an example). A spin chain is attached to every pair of the two nearest neighbors along the third dimension in the 3D Ising lattice. For simplicity, Figure 5 just illustrates a unit cell of the 3D cubic Ising model, with the attachments of four spin chains. One spin located at the 3D Ising lattice is attached by two spin chains (however, it should be divided by a factor 2 to avoid over-account). Figure 5 is obtained directly from Figure 3, by a mapping shown in Figure 4. Figure 6 shows that a spin on the 3D Ising model is connected with eight edges, and it has eight nearest neighbors (six from the original 3D Ising lattice and two from the spin chains). This explains the origin of the additional dimension attached to the 3D Ising model. Therefore, the number of the first neighboring sites of the 3D Ising model should be equal to that of the "big" lattice, which is 8 . This answers why the first term in the low-temperature expansion of the spontaneous magnetization of the 3D Ising model should be $-6 x^{8}$ [3], which differs from the leading term $-2 x^{6}$ of conventional low-temperature expansion.

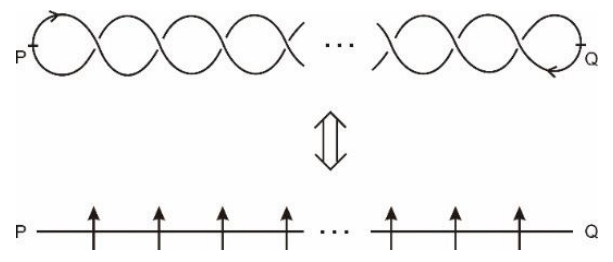

Figure 4. A braid is mapped to a spin-chain lattice. 


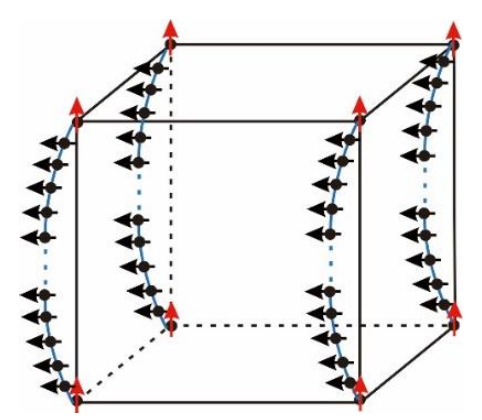

Figure 5. A unit cell of the 3D cubic Ising lattice with an additional "imagine" Ising spin lattice. The "imagine" spin chains, formed by the mapping from the nontrivial knots (nonlinear terms) of $\Gamma$-matrices of the transfer matrices, are attached to every pair of the two nearest neighboring lattice sites along the third dimension of the 3D Ising lattice. A "big" spin lattice is constructed by the original 3D Ising lattice (red arrows with black lines) and the "imagine" spin chains (black arrows with blue curves).

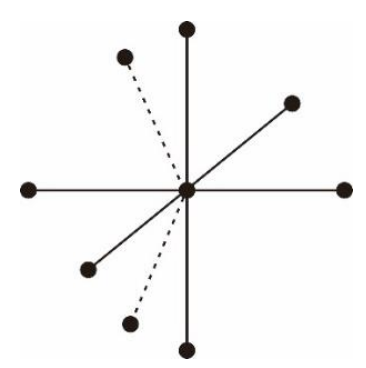

Figure 6. A spin located in a lattice point of a "big" spin lattice has the eight nearest neighbors, six from the original 3D Ising lattice (solid lines) and two from the "imagine" spin chains (dashed lines).

Clearly, the "big" spin lattice constructed above differs from a 4D lattice. In the "big" lattice shown in Figure 5, the lattice points in a spin chain are not connected with those in its neighboring spin chains. If the lattice points in a spin chain are connected with those in their six neighboring spin chains, this will form a full 4D lattice. The "big" lattice is denser than the original 3D lattice but more discrete than the 4D lattice. After such a mapping, our spins (including the original ones and the "imagine" ones) do not occupy all the lattice points of the 4D lattice, namely, the 4D lattice is not fully occupied. This character results in the physical properties of our 3D Ising model being in between those of the "pure" 3D (without consideration of the long-range entanglement) and 4D Ising lattices (see Remarks 3 and 4).

Zhang, Suzuki and March proved four theorems (Trace Invariance Theorem, Linearization Theorem, Local Transformation Theorem, Commutation Theorem) by a Clifford algebra approach [7], verifying the correctness of Zhang's exact solution for the ferromagnetic (or antiferromagnetic without frustration) 3D Ising model in the zero magnetic field. In what follows, we briefly introduce the Zhang-Suzuki-March approach [7]. At first, the $3 \mathrm{D}$ Ising model is extended to be the $(3+1) \mathrm{D}$ one and divided to many quasi-2D Ising models. Then, the nonlinear terms in the transfer matrices are linearized, while the Hilbert space is split. This corresponds to cutting the spin chains in Figure 5 and rearranging the spins in the larger space. Finally, a local transformation is performed, which is represented by a rotation in $4 \mathrm{D}$ or $(3+1) \mathrm{D}$ space, and serves to introduce an additional dimension with "spins" interacting with each other, while three topological phases are generated on quaternionic eigenvectors $\psi_{(3+1) D}^{\prime}=w_{x} \psi_{2 D}^{i} \vec{i}+w_{y} \psi_{2 D}^{j} \vec{j}+w_{z} \psi_{2 D}^{k} \vec{k}[3,4,7]$ (see Equation (33) of [3] for details). This results in the desired solution, a $(3+1) \mathrm{D}$ Ising lattice with the topological phases acting as a projection from $4 \mathrm{D}$ to $3 \mathrm{D}$. The $(3+1) \mathrm{D}$ Ising lattice is schematically illustrated in Figure 7, which is obtained by performing a local 
transformation on Figure 5. Indeed, the number of the first neighboring sites of the $(3+1) \mathrm{D}$ Ising lattice equals 8.

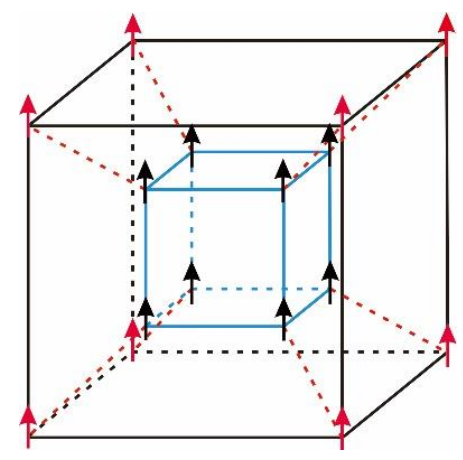

Figure 7. A unit cell of the $(3+1) \mathrm{D}$ hypercubic Ising lattice in which the spins on the original 3D Ising lattice (black lines) are represented by red arrows and the spins in the high dimensional lattice (blue lines) are represented by black arrows. The red dashed lines connect the vertexes in the two cubes, representing a hypercube in 4D.

\subsection{Viewing the 3D Ising Model from an Angle of the Topological Structures}

On the other hand, one can view the 3D Ising model from another angle of the topological structures. As revealed in $[3,4,7,10]$, an intrinsic nontrivial topological structure of the transfer matrices exists in the representation of $\Gamma$-matrices, due to the long-range effective interaction (entanglement) between the spins in the 3D Ising lattice. In addition, all the Ising spins located in the 3D lattice can be mapped to an "imagine" nontrivial topological structure. Both the nontrivial topological structures join together to form a "big" topological structure in higher dimensional space (i.e., $(3+1) \mathrm{D}$ or $4 \mathrm{D})$. Figure 8 shows an example of knots in the $(3+1) D$ dimensions, which is constructed by crossings mapped from spins in a unit cell of the 3D Ising lattice and braids originating from the nontrivial topological structure corresponding to the nonlinear terms in the transfer matrices. Such a construction can be extended to all the 3D Ising lattice to cover the whole $(3+1)$ dimensions. The "big" topological structure consists of an original topological structure (braids, corresponding to the nonlinear terms in the transfer matrices) and an "imagine" topological structure (formed by the mapping from the spin lattice, corresponding to all the linear terms in the transfer matrices).

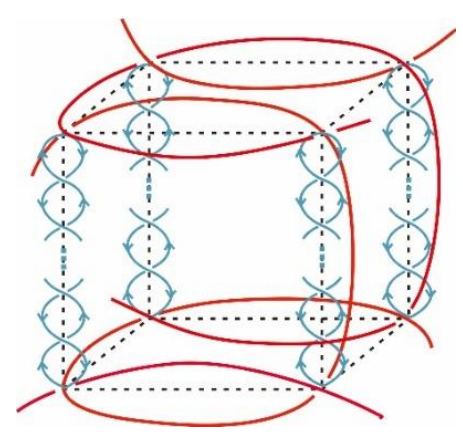

Figure 8. An example of knots constructed from two parts, in which some crossings (red curves) are mapped from spins in a unit cell (black dashed lines) of the 3D Ising lattice, and some crossings (blue curves) are attached braids that come from the nontrivial topological structure, originating from the nonlinear terms of the transfer matrices.

Following the Zhang-Suzuki-March approach [7], the knots can be trivialized by performing the local transformation naturally in the $(3+1) \mathrm{D}$ or $4 \mathrm{D}$ space. The local transformation transforms the braids (blue curves) in Figure 8 to the crossings in the higher dimensional lattice, as the blue curves shown in Figure 9. This is consistent with the 
topology theory that any knots in $4 \mathrm{D}$ space can be opened. In Figure 9, one can view the "big" topological structure as: the original topological structure evaluates with time to form the "big" topological structure in $(3+1) \mathrm{D}$ or $4 \mathrm{D}$, in which all the crossings may be compensated and/or smoothed.

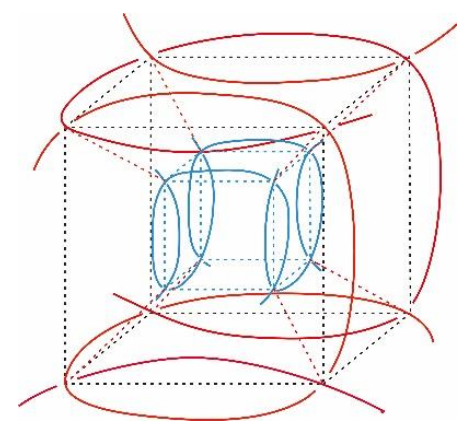

Figure 9. An example of knots with crossings located on a unit cell of the $(3+1) \mathrm{D}$ hypercubic Ising lattice. The black and blue dashed lines represent two cubes, while the red dashed lines connect the vertexes of the two cubes, together representing a hypercube. The crossings corresponding to the vertexes in the black cube lattice are represented by red solid curves, while the crossings corresponding to the vertexes in the blue cube lattice are represented by blue solid curves. All the crossings form a "big" topological structure that is trivialized naturally.

\subsection{A Theorem for Zhang's Conjectures}

From these observations, we see the following theorem.

Theorem 1. The 3D Ising model $\left(I_{3 D}\right)$ in the zero external magnetic field can be mapped to either a $(3+1) D$ Ising spin lattice or a trivialized topological structure in the $(3+1) D$ or $4 D$ space. Namely, starting from the definition in [10]:

$$
\mathrm{Cl}\left(I_{3 D}\right) \oplus \overline{\mathrm{Cl}\left(I_{3 D}\right)} \Leftrightarrow K / C\left(I_{3 D}\right) \longrightarrow \mathrm{Cl}^{*}\left(I_{3 D}\right)
$$

we have the following mappings:

(1) $\mathrm{Cl}^{*}\left(I_{3 D}\right)=\mathrm{Cl}\left(I_{3 D} \circ S^{1}\right) \Leftrightarrow \mathrm{Cl}\left(I_{(3+1) D}\right)$;

(2) $K / C\left(I_{3 D}\right) \Leftrightarrow K / C\left(I_{3 D} \circ S^{1}\right) \Leftrightarrow K / C\left(I_{(3+1) D}\right)$.

Additionally, the two mappings are equivalent and thus we have: $C l\left(I_{(3+1) D}\right) \Leftrightarrow K / C\left(I_{(3+1) D}\right)$.

Remark 2. The exact expression for the spontaneous magnetization of the simple cubic lattices is given in Equation (101) or (102) in [3], while its low-temperature expansion with the leading term $-6 \times{ }^{8}$ is represented in Equation (103) in [3].

Remark 3. The critical exponents of the 3D Ising model are in between the values from calculations for the 3D Iisng lattice taking into account only the contributions of the local environments of spin alignments and those (mean-field theory) of the $4 D$ Ising lattice. The critical exponents of the $3 D$ Ising model are determined to be exactly $\alpha=0, \beta=3 / 8, \gamma=5 / 4, \delta=13 / 3, \eta=1 / 8$ and $v=2 / 3[3,7,9]$.

Remark 4. In order to calculate the physical properties of the 3D Ising model by computer simulations, one must calculate those of the $(3+1) D$ lattice with spins located at the original $3 D$ Ising lattice and at the spin chains that are attached on every pair of two neighboring lattice sites along the third dimension of the 3D Ising lattice, plus the local transformation with the topological phases on the eigenvectors, which projects the system from $(3+1) D$ to $3 D$.

Remark 5. Either the braid in Figure 3 or the spin chain in Figure 5 represents a long-range effective interaction between spins in a plane, an entanglement involved with all spins in the 
plane, and a nontrivial topological structure $[3,4,7,10]$, which also exists in the spin-glass $3 D$ Ising model [8]. It helps us to figure out that the absolute minimum core (AMC) model exists, which consists of a spin-glass 2D Ising model interacting with its nearest neighboring plane [8]. It verifies that the lower bound of the computational complexity of a spin-glass 3D Ising model is controlled by the upper bound of the computational complexity of the AMC model, which is in subexponential time, superpolynomial.

The statements above describe a scenario, giving a simplified and concise version (actually, a schematic illustration) of proofs of Zhang's conjectures 1 and 2. This schematic proof is consistent with the procedures developed in our previous work $[7,10]$. In the Clifford algebraic approach developed in [7], the additional dimension is introduced by adding the direct product of many unit matrices, by performing the time average and by performing a local transformation (a rotation in local coordinates) via a transformation matrix. In the topological approach based on the Riemann-Hilbert problem [10], the additional dimension is introduced by realizing the knots/links of the 3D Ising model on Riemann surfaces, and by performing the monoidal transformation in a 4D Riemann manifold. Therefore, the 3D Ising model provides us a platform for a deep understanding of the spontaneous emergence of the additional dimension (i.e., time), the construction of the $(3+1) \mathrm{D}$ framework for the space-time lattice, the mathematical base of topological quantum statistical mechanics and even the connection between algebra, topology, and geometry (see Section 7 for a detailed description). In the remainder of this paper, we shall focus our interest on the application of the Riemann-Hilbert problem, to solve Zhang's conjecture 2 of the 3D Ising model.

\section{Representation of Fundamental Group}

In this section, we recall the basic facts of the representations of the fundamental group for the ferromagnetic 3D Ising model in a zero magnetic field and construct the representations of knots [10].

The trivial topological states are defined as a circle (or an interval) and a knot of type I, corresponding to the linear terms in the transfer matrices $\mathbf{V}_{\mathbf{1}}$ and $\mathbf{V}_{\mathbf{2}}$ [10]. The nontrivial topological states are defined as a braid with many crossings, called the basic form of type II [10]. The nontrivial topological states for the ferromagnetic 3D Ising model are constructed by combination of the knot $\gamma$ of the $3 \mathrm{D}$ lattice $\mathrm{Z}_{3}$ and the trivial and nontrivial topological states. For a detailed description of the topological structures, please refer to Figures 1-4 of our preceding paper [10] and also Section 2 above.

We recall the basic facts on the representation of the fundamental group [10]. Let $M$ be a manifold, $p_{0} \in M$ and let $\pi_{1}\left(M, p_{0}\right)$ be the fundamental group of $M$ based at $p_{0}$. Consider a monodromy representation: $\rho: \pi_{1}\left(M, p_{0}\right) \rightarrow G L(M, C)$. We choose a vector space $V_{M}$, which represents the representation:

$$
\gamma^{*} \mathrm{f}=\rho(\gamma) f
$$

where $\rho(\gamma)$ is called the multiplicative factor with regular singularities at $a_{j}(j=1,2, \ldots$ $\ldots$, M) for any closed path $\gamma$ (Röhrl Theorem [21]).

According to Theorem II in the preceding paper [10], the Riemann-Hilbert problem is applied for the representation on the ferromagnetic 3D Ising model in the zero magnetic field. For the representation of knots/links for the ferromagnetic 3D Ising model in the zero magnetic field, we consider 3D lattices and their periodic compactification, which is denoted by ${ }^{-} \mathrm{C} u \bar{b}$. We describe the fundamental group. It is generated by $\gamma_{1}, \gamma_{\overline{1}}, \ldots, \gamma_{m}, \gamma_{\bar{m}}$, which constitute the big circles. They are generated by $[10] \Gamma_{1}, \bar{\Gamma}_{1}, \Gamma_{2}, \bar{\Gamma}_{2}, \ldots, \Gamma_{m}, \bar{\Gamma}_{m}$. The lattice $\mathrm{Cl}(\mathrm{Z})$ is given by:

$$
\left\{K_{1} \Gamma_{1}+\bar{K}_{1} \bar{\Gamma}_{1}+\ldots+K_{m} \Gamma_{m}+\bar{K}_{m} \bar{\Gamma}_{m} \mid K_{i}, \bar{K}_{i} \in Z\right\}
$$

It is clear that $\mathrm{Cl}\left(I_{3 D}\right)$ is generated by $\mathrm{Cl}(\mathrm{Z})$. 
Next we pay some attentions to the singularities of the high-temperature expansions of the free energy f. Perk argued (see Equations (6) and (7) in [25]) that $\beta \mathrm{f}$ has an absolutely convergent series $\left(\beta=1 /\left(\mathrm{k}_{\mathrm{B}} \mathrm{T}\right)\right)$, uniformly convergent in the thermodynamic limit, so that

$$
\beta \mathrm{f}=\sum_{i=0}^{\infty} a_{i} \beta^{i},|\beta|<r
$$

and that

$$
\mathrm{f}=\frac{a_{0}}{\beta}+\sum_{i=1}^{\infty} a_{i} \beta^{i-1}, 0<|\beta|<r
$$

is a convergent Laurent series. The question is whether $\mathrm{f}$ is totally equivalent to $\beta \mathrm{f}$, especially at $\beta=0$. Perk argued that the pole at $\beta=0$ has no significance [25]. Actually, there are two kinds of singularities in the 3D Ising model at the zero magnetic field. One is the singularity (pole) at $\beta=0$, and another is the singularity caused by crossings in the nontrivial knots. For the singularity at $\beta=0$, when we solve the Riemann-Hilbert problem $[21,22]$ for $\mathrm{f}$ with a singularity and $\beta \mathrm{f}$ without a singularity, different results will be obtained upon a monodromy representation:

$$
\begin{aligned}
& \frac{d w}{d \beta}=f w=\left(\frac{a_{0}}{\beta}+\ldots\right) w \\
& \frac{d w^{\prime}}{d \beta}=\beta f w^{\prime}=\left(a_{0}+\ldots\right) w \prime
\end{aligned}
$$

Note that a transformation $\beta \rightarrow$ it would transform the above equations to Schrödinger equations. Clearly, $\frac{d w}{d t} \neq \frac{d w^{\prime}}{d t}$ at $\beta=0$. Thus, with the framework of quantum mechanics, we have $w \neq w^{\prime} \Leftrightarrow E_{n} \neq E_{n}^{\prime}$ for $\beta>0$. In addition, the key here is that other singularities corresponding to crossings in $\mathrm{f}$ (and also $\beta \mathrm{f}$ ) exist for $\beta>0$, which are not taken into account in the conventional high-temperature expansions for $\beta \mathrm{f}$. The correct formula for high-temperature expansions of the free energy $\mathrm{f}$ (and also $\beta \mathrm{f}$ ) must account for the contributions of the singularities of these crossings.

Remark 6. The Röhrl Theorem [21] provides the possibility of the existence of a multi-valued function with regular singularities for a given monodromy representation. For the ferromagnetic 3D Ising model in the zero magnetic field, it consists of the generation of topological phases on the eigenvectors and the topological phase transition at/near the infinite temperature. At/near the infinite temperature, the 3D Ising system has no nontrivial topological structure since the interactions are comparatively small compared with the infinite temperature, and the conventional high-temperature expansions works well in this region. However, at the finite temperature, the interactions become dominant, introducing the nontrivial topological structure that also contributes to the partition function and the physical properties, where the conventional high-temperature expansion is lost. Indeed, a multi-valued function exists at/near the infinite temperature for the ferromagnetic 3D Ising model in the zero external magnetic field, which results in different functions at the finite temperature.

\section{Vertex Operators of Knot Type and the Corresponding Description of the Representation}

In this section, we introduce a concept of vertex operators of knot types for the ferromagnetic 3D Ising model in the zero external magnetic field, which is the analogy of the vertex operators in the Conformal Field Theory [26-39]. We can prove the following theorem:

Theorem 2. The partition function of the ferromagnetic 3D Ising model in the zero magnetic field can be described in terms of the product of vertex operators.

The proof of Theorem 2 is represented in Appendix A.

The partition function of the ferromagnetic 3D Ising model in the zero magnetic field can be described in terms of the transfer matrices, in which the linear and nonlinear terms correspond to the knots of type I and type II [10]. In Appendix A, the basic forms of knot 
types I and II are described in terms of vertex operators. Thus, the partition function of the ferromagnetic 3D Ising model in the zero magnetic field can also be described in terms of vertex operators.

Zhang and March [40] suggested that the quaternion-based functions developed in [3] for the ferromagnetic 3D Ising model in the zero external magnetic field can be employed to investigate the conformal invariance in dimensions higher than two. We summarize here the procedure Zhang and March developed in [40] for treating the 3D conformal field theory: (1) introducing an additional dimension to construct a $(3+1) \mathrm{D}$ framework to form the quaternionic coordinates; (2) performing a transformation, as a rotation in $(3+1)$-dimensions, to represent states and operators in the $(3+1) \mathrm{D}$ complexified quaternionic Hilbert space; (3) introducing complex weight factors as topological phase factors $\mathrm{w}_{\mathrm{i}}$, for smoothing knots/crossings; (4) decomposition of 3D conformal blocks to 2D conformal ones; (5) dealing with 2D conformal blocks in each complex plane of quaternionic coordinates of the complexified quaternionic Hilbert space; and (6) accounting the summation i of $2 \mathrm{D}$ conformal blocks in three complex planes and considering the contributions of the phase factors $\mathrm{w}_{\mathrm{i}}$.

\section{Flat Vector Bundle and Analytic Realization of the Integrable System of the 3D Ising Model}

In this section, a flat vector bundle is introduced from the solution of the RiemannHilbert problem and the integrable system of the ferromagnetic 3D Ising model in the zero magnetic field is shown to be equivalent to the flat vector bundle. By use of the identification, for example, the Berry phase [41,42] and the Gauss-Bonnet-Chern formula [43,44]. The differential geometric structure of the 3D Ising model is discussed.

\subsection{Flat Vector Bundle of the Monodromy Representation}

In a well-known manner, we can construct a flat vector bundle [22,45] from the solution of the Riemann-Hilbert problem. We consider the representation [10]:

$$
\widetilde{\rho}: \pi_{1}\left(R_{g}-\left\{a_{1}, \ldots, a_{N}\right\}\right) \otimes C l\left(I_{3 D}\right) \rightarrow C L^{*}\left(I_{3 D}\right)
$$

A system of the local coordinate system $\left\{U_{\alpha}\right\}$ of $R_{g}$ is made in the following manner. At first we choose local coordinates with the origin $a_{1}, \ldots \ldots a_{N}$, which are denoted by $U_{1}, \ldots \ldots U_{N}$. Next, the finite local coordinate neighborhood is added to make a local coordinate system $\left\{U_{\alpha}\right\}$. Choosing a base point $P_{0}$, we assume that $P_{0} \in U_{0}$ with some $U_{0}$. We choose a point $P_{\alpha} \in U_{\alpha}$ (here we assume that $P_{\alpha} \neq a_{j}\left(\forall_{j}\right)$ ).

We choose a path $\gamma_{j}$ which joints $P_{0}$ and $P_{j}$. It is assumed that $\gamma_{j} \nexists a_{j}\left(\forall_{j}\right)$. A vector bundle can be constructed, as shown in Figure 10. We consider $U_{\alpha}, U_{\beta}$ with $U_{\alpha} \cap U_{\beta} \neq \varnothing$ and choose $\forall P \in U_{\alpha} \cap U_{\beta}$. This makes a path $\gamma_{\alpha \beta}(P)$ which joints with $P_{\alpha}$ and $P_{\beta}$ and $P \in \gamma_{\alpha \beta}(P)$.

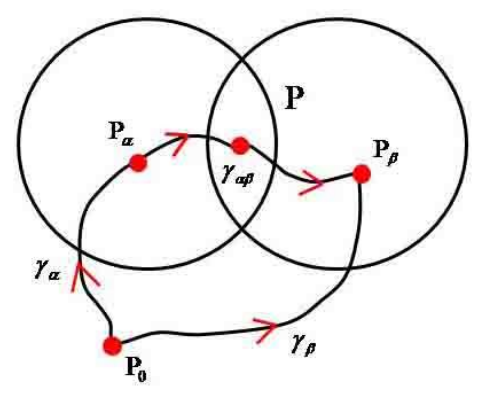

Figure 10. Construct a vector bundle.

It leads to a closed path, $\widetilde{\gamma}_{\alpha \beta}=\gamma_{\alpha} \gamma_{\alpha \beta} \circ \gamma_{\beta}^{-1}$, here $\gamma_{\beta}^{-1}$ means the path from $P_{\beta}$ to $P_{0}$. 
Putting $C_{\alpha \beta}(P)=\widetilde{\rho}\left(\widetilde{\gamma}_{\alpha \beta}\right),\left(P \in U_{\alpha} \cap U_{\beta}\right)$, it determines an element in $C L^{*}\left(I_{3 D}\right)$ (see Equation (10)), we see that

$$
\left\{\begin{array}{l}
C_{\alpha \beta} \cdot C_{\beta \gamma}=C_{\alpha \gamma} \\
C_{\alpha \beta} \cdot C_{\beta \alpha}=1
\end{array}\right.
$$

This results in a flat vector bundle $E_{\widetilde{\rho}}$, which is called the vector bundle of the monodromy representation. For details, please refer to [21,22].

Some basic facts on the vector bundle are stated as follows [21,22]:

(1) $C_{\alpha \beta}$ is a constant matrix $\left(\operatorname{det}\left(C^{\alpha \beta}\right) \neq 0\right)$. Hence $E_{\widetilde{\rho}}$ becomes a locally trivial vector bundle.

(2) Paths $\gamma_{\alpha}, \gamma_{\alpha \beta}$ are chosen not uniquely. The different choices give equivalent vector bundles.

(3) A solution $\mathrm{W}$ of the Riemann-Hilbert problem gives rise to a meromorphic section $\phi$ of $E_{\tilde{\rho}}$.

The converse is also true. The correspondence is given as follows:

Choosing $\mathrm{W}$ on $U_{0}$ and making analytic continuation along $\gamma_{\alpha}$, we define $\phi_{\alpha}=\gamma_{\alpha}^{*}(\widetilde{W})$. Then it is seen that $\varphi_{\alpha}=C_{\alpha \beta} \varphi_{\beta}$; hence, one has $\varphi \in \Gamma\left(R_{g}-\left\{a_{1}, \ldots, a_{N}\right\}, E_{\rho}\right)$.

(4) A solution $W$ of the Riemann-Hilbert problem gives a flat connection $\omega$ of $E_{\widetilde{\rho}}$ by:

$$
\left\{\begin{array}{l}
\omega=\operatorname{dlog} \widetilde{W} \\
d \omega=\omega \wedge \omega
\end{array}\right.
$$

Note that the connection identifies the parallel displacement of a tangent vector in one tangent space to a tangent vector in a different tangent space in general curved spaces.

\subsection{Equivalence of the Integrable Systems}

We can treat the ferromagnetic 3D Ising model in the zero external magnetic field as integrable systems. We can prove that both the integrable systems which are given in the description of $\Gamma$-matrices for the partition function and the flat vector bundle are equivalent.

Theorem 3. The integrable system of the 3D Ising model in the zero magnetic field in the description of $\Gamma$-matrices for the partition function is equivalent to the flat vector bundle.

Proof of Theorem 3: The equivalence between the description with $\Gamma$-matrices of the partition function and the flat vector bundle can be constructed as follows:

(1) The partition function described in terms of $\Gamma$-matrices can be represented in the topological structures by the $\mathrm{K} / \mathrm{C}$ algebra [10]. We have the representations of the fundamental group of a manifold $\mathrm{M}$, in order to construct the representation of knots.

(2) The Riemann-Hilbert problem is applied for the representation on the ferromagnetic 3D Ising model through the monodromy representations (Röhrl Theorem [21]). The Riemann-Hilbert problem asks for the solution of a differential equation which admits regular singularities at a set of descrete points on a manifold $M$. If $\Phi$ is a $\rho$-multiplicative function, we will obtain the following matrix valued meromorphic 1-form $\omega$ on the manifold $M[21,22]$ :

$$
\omega=\Phi^{-1} d \Phi
$$

It is remarked that $\omega$ satisfies the following integrability condition [21,22]:

$$
d \omega-\omega \wedge \omega=0
$$

Conversely, taking a 1-form $\omega$ at the regular singularities on $M$, with the condition Equation (14) and the differential Equation (13), we obtain a multivalued function $\Phi$. Equation (14) is just the same as Equation (12) for the definition of a flat connection $\omega$. 
(3) It is seen that Equation (13) can be written as:

$$
d \Phi=\Phi \omega
$$

It is remarkable that Equation (15) has a form that is very analogous to the famous Schrödinger equation. In this way, the solution of a model in quantum mechanics can be associated with the flat connection in geometry. Although, in quantum statistical mechanics we are interested in the thermodynamical properties with the ensemble average for the stationary Schrödinger equation $H \Psi=E \Psi$, where $H$ is the Hamiltonian, $E$ is the eigenvalues of the energy, and $\Psi$ is the eigenvectors (wavefunctions) of the physical system, the wavefunctions must also satisfy the Schrödinger equation:

$$
i \hbar \frac{\partial \Psi}{\partial t}=H \Psi
$$

Indeed, the solution of the physical system we are studying must satisfy the differential Equation (16) (the Schrödinger equation). In our previous work $[3,4,7,10]$, we have proven that the time average must be taken into account, while quaternionic eigenvectors are constructed for the 3D Ising model. It indicates that time must be considered to construct the $(3+1) \mathrm{D}$ framework to study the evolution of the 3D Ising model. It is understood that the many-body interactions in the 3D Ising spin lattice make the wavefunctions composed of space and time coordinates, which together form quaternionic eigenvectors. In this sense, the wavefunctions are not stationary but are dynamical ones.

(4) From Equation (13), performing integral and differential processes results in the following relation:

$$
\omega=d \log \Phi
$$

Again, it is consistent with Equation (12) for the definition for a flat connection $\omega$ if one uses W to replace $\Phi$. Clearly, from the definition above, $W$ is a solution of the Riemann-Hilbert problem with the monodromy representation. Note that this solution can be seen as the gauge factor generated in the wavefunction $\Phi^{\prime}$ after a gauge transformation, which corresponds to the monodromy representation. It gives rise to topological/geometrical phase factors on the eigenvectors with renormalization of the 3D Ising model, derived from the curvature according to the Gauss-Bonnet-Chern formula (see the next section).

(5) The vertex operators are introduced to establish the equivalence between the knot structures in the descriptions of the $\mathrm{K} / \mathrm{C}$ algebra and vertex operators for the partition function (see Section 4 and Appendix A, and also [10]).

(6) The flat vector bundle is introduced to establish the equivalence between the solutions of a model in quantum statistical mechanics and a differential equation which admits regular singularities on a manifold $M$ (see the detailed descriptions in this section). Taking $\mathrm{W}$ has regular singularities into account, we can treat it as the Berry phase [41,42].

\section{Renormalization of the 3D Ising Model}

In this section, we shall construct renormalization of the ferromagnetic 3D Ising model in the zero external magnetic field and show that the model can be realized as a free model on the manifold with nontrivial curvature.

\subsection{Gauss-Bonnet Formula}

We recall the Gauss-Bonnet formula $[43,44]$ which derives the curvature from the topological nontrivial field (Berry phase [41,42]):

$$
\int_{M} K d A+\int_{\partial M} k_{g} d s=2 \pi \chi(M)
$$


where $d A$ is the element of the area of the surface, and $d s$ is the line element along the boundary of $M$. Here, $\chi(M)$ is the Euler characteristic. If the boundary $\partial \mathrm{M}$ is piecewise smooth, we will interpret the second integral $\left.\int_{\partial M} k_{g} d s\right)$ as the sum of the corresponding integrals along the smooth portions of the boundary, plus the sum of the angles by which the smooth portions turn at the corners of the boundary. Chern discovered a higherdimensional analogue of the original Gauss-Bonnet theorem for 2D manifolds [45]:

$$
\int_{M} \operatorname{Pf}(\Omega) d M=(2 \pi)^{m} \chi(M)
$$

where $M$ is a $2 \mathrm{~m}$-dimensional Riemann manifold, $\Omega$ is the curvature of $M$ given via the Levi-Civita connection, the Pfaffian of $\Omega$ is defined by $\operatorname{Pf}(\Omega)=[\operatorname{det}(\Omega)]^{1 / 2}$ and $\chi(M)$ is the generalized Euler characteristic, that is, Chern number for the class $\mathrm{m}$.

We take a Riemann surface and take out finite points $a_{1}, \ldots \ldots a_{N}$. It is assumed that a matrix valued meromorphic 1 -form $\omega$ exists, which admits the residue $\beta_{j}$ at each point $a_{j}$ $(j=1,2 \ldots \ldots N)$ :

$$
\omega=\frac{\beta_{\mathrm{j}}}{\mathrm{z}-a_{j}} d z+\ldots \ldots
$$

This implies that a holomorphic function with regular singularities exists:

$$
\mathrm{F}(\mathrm{z})=\left(z-a_{j}\right)^{2 \pi i \beta_{j}}\left[\alpha_{0}+\alpha_{j}\left(z-a_{j}\right)+\ldots \ldots\right]
$$

Hence there is a topological trivial line bundle $\pi: E_{\rho} \rightarrow R$. Next making "renormalization" of the singularities, we avoid the singularities, having the nontrivial curvature. This is nothing but the Gauss-Bonnet formula.

As we have seen in Sections 4 and 5, we can obtain a section

$$
\varphi \in \Gamma\left(R_{g}-\left\{a_{1}, \ldots, a_{N}\right\}, E_{\rho}\right)
$$

from F. Putting

$$
\varphi_{j}=\left\{\begin{array}{lcc}
\left(\mathrm{z}-\mathrm{a}_{\mathrm{j}}\right)^{2 \pi \mathrm{i} \beta j} \cdot \varphi & \text { on } & U_{j} \\
\varphi & \text { on } & \text { otherwise }
\end{array}\right.
$$

We have the "renormalized section" $\varphi=\left\{\varphi_{j}\right\}$. From this we can obtain the renormalized line bundle: $E_{\gamma}=\left\{\varphi_{i j}\right.$ on $\left.U_{i} \cap U_{j}\right\}$ :

$$
\varphi_{i j}=\varphi_{i}^{-1} \varphi_{j}
$$

Then $\left\{\omega_{i}\right\}$ becomes a section of $E_{\gamma}$. Next the curvature is given by introducing the metric of $E_{\gamma}: a=\left\{a_{j} \mid a_{j}>0\right\}$ is called metric of $E_{\gamma}$, when $a_{j}=\left|\varphi_{i j}\right|^{2} a_{i}$ on $U_{i} \cap U_{j}$.

It is seen that $\phi=a_{i}\left|\varphi_{j}\right|^{2}$ on $U_{j}$ becomes a global function on the Riemann surface. We calculate the curvature $\partial \bar{\partial} \log \phi[22,43-54]$ :

$$
\partial \bar{\partial} \log \phi=\partial \bar{\partial} \log a_{i}+\partial \bar{\partial} \log \left|\varphi_{j}\right|^{2}=\partial \bar{\partial} \log a_{i}+d\left(\bar{\partial} \log \left|\varphi_{j}\right|^{2}\right)
$$

Integrating it on $R-\left\{U_{\varepsilon}\left(a_{1}\right) \cup \ldots \ldots \cup U_{\varepsilon}\left(a_{m}\right)\right\},\left(=R_{\varepsilon}\right)$

$$
\iint_{R_{\varepsilon}} \partial \bar{\partial} \log \phi=\iint_{R_{\varepsilon}} \partial \bar{\partial} \log a_{i}+\iint_{R_{\varepsilon}} d\left(\bar{\partial} \log |\varphi|^{2}\right)
$$

The second term becomes:

$$
\int_{\partial R_{\varepsilon}} \bar{\partial} \log \left|\varphi_{i}\right|^{2}=\int_{\partial R_{\varepsilon}} \frac{\bar{\partial} \bar{\varphi}_{i}}{\bar{\varphi}_{i}}
$$


Tending $\varepsilon \rightarrow 0$, we see:

$$
\lim _{\varepsilon \rightarrow 0} \iint_{R_{\varepsilon}} \partial \bar{\partial} \log |\phi|=0
$$

This results in the so-called Gauss-Bonnet-Chern formula [22,43-51]:

$$
\sum_{i=1}^{N} \operatorname{Res}_{i} \varphi=\frac{1}{2 \pi i} \iint_{R} \partial \bar{\partial} \log a_{i}=c_{1}(F)
$$

This is one of the basic approaches to the renormalization theory. By the procedures above, we have taken off nontrivial knots in a higher dimensional space. $c_{1}(F)$ is the Chern number of the first class, while Res denotes the residue.

Remark 7. The Gauss-Bonnrt-Chern formula is consistent with Fuchs relation [22,55-57] for solutions of a differential equation which admits regular singularities on a Riemann surface. Suzuki [22] derived a generalized Fuchs relation, in which the Chern number of the first class is related with the order $n$ of differential equations, the number $m$ of singularities, and the genus $g$ of the manifold by the following formula: $c_{1}=\frac{n(n-1)}{2}(m+2(g-1))$. For the present case, we may set $n=2, m=4, g=0$ for $c_{1}=2$.

\subsection{Gauss-Bonnet-Chern Formula for the Ferromagnetic 3D Ising Model}

Applying the idea to the ferromagnetic 3D Ising model at the zero magnetic field, we obtain the Gauss-Bonnet-Chern formula [43-50]. In consideration of the representation:

$$
\rho_{0}: \mathrm{KL}(3 \mathrm{D}) \mathrm{Cl}\left(I_{3 D}\right) \longrightarrow \mathrm{Cl}^{*}\left(I_{3 D}\right)
$$

we have

$$
\widetilde{\rho}: \pi_{1}\left(R_{g}-\left\{a_{1}, \ldots, a_{N}\right\}\right) \otimes C l\left(I_{3 D}\right) \rightarrow C L^{*}\left(I_{3 D}\right)
$$

Solving the Riemann-Hilbert problem, we have the solution $F$ which has a regular singularity at each $a_{i}(i=1, \ldots \ldots N)$. Making the vector bundle of the representation $\mathrm{E}_{\widetilde{\rho}}$ and the section $\hat{F}$ of $\mathrm{E}_{\widetilde{\rho}}$ corresponding to $F$, we can obtain the Gauss-Bonnet-Chern formula for the representation (see Appendix $B$ for details).

From the results obtained above, the 3D Ising model with nontrivial topological structures can be realized as a trivial model on a nontrivial topological manifold. Indeed, a map between the trivial manifold and the nontrivial manifold exists, which correlates the Chern numbers of the two manifolds with winding numbers. According to algebraic topology and differential geometry [41-51], one may expect to fix the winding numbers and topological phases for the ferromagnetic 3D Ising model in the zero magnetic field. Its space is extended to the $(3+1)$ dimensions, which can be split into three subspaces in $(1+1)$ dimensions for the 2D Ising planes [43-51,58-61]. The characteristic of the $(3+1) \mathrm{D}$ space equals the sum of the characteristics of three $(1+1)$ dimensional subspaces [43-51,58-61]. For a representation on $\rho$, we can calculate the winding number for each "coordinate" $\vec{i}, \vec{j}$, $\vec{k}$ in the quaternionic space of the eigenvectors $\psi_{(3+1) D}^{\prime}=w_{x} \psi_{2 D}^{i} \vec{i}+w_{y} \psi_{2 D}^{j} \vec{j}+w_{z} \psi_{2 D}^{k} \vec{k}$ of the ferromagnetic 3D Ising model [3,4,7] (see Equation (33) of [3] for details), which are denoted by $\mathrm{n}_{1}, \mathrm{n}_{2}$, and $\mathrm{n}_{3}$. Note that the quaternion may be used to represent a "vector" in a 3D space, although the quaternionic eigenvectors themselves are not vectors. We can perform the path integrals along the three "coordinates" and then sum the results. One of the three "coordinates" of the quaternionic space corresponds to the 2D Ising character with the winding number $\mathrm{n}_{1}=1$ (for $2 \mathrm{D}$ rotations of Ising spins in a plane), while the other two "coordinates" represent the 3D Ising character with the winding numbers $\mathrm{n}_{2}=\mathrm{n}_{3}=2$ (in consideration of $3 \mathrm{D}$ rotations of Ising spins with $Z_{2}$ symmetry). Then we obtain the following formula:

$$
n_{1} \kappa_{1}+n_{2} \kappa_{2}+n_{3} \kappa_{3}=c_{1}\left(E_{\rho}^{\prime}\right)
$$


where $c_{1}\left(E_{\rho}^{\prime}\right)$ is the characteristic class of $E_{\rho}^{\prime}$. Note that the Chern number $c_{1}\left(E_{\rho}^{\prime}\right)$ of the first class corresponds to the 2D manifold, which equals to two. This is because although the 3D Iding model is on a 4D Riemann manifold as a whole (in $(3+1) \mathrm{D}$ spacetime), each coordinate $\vec{i}, \vec{j}, \vec{k}$ corresponds to a 2D manifold (Riemann surface), and the $4 \mathrm{D}$ Riemann manifold is constructed by many $2 \mathrm{D}$ manifolds, as revealed already in our previous work [7]. Meanwhile, $\kappa_{1}, \kappa_{2}$, and $\kappa_{3}$ are the characteristic class for the three "coordinates" (can be seen as three sub-manifolds) of the quaternionic space. Then we have

$$
n_{1} \phi_{x}+n_{2} \phi_{y}+n_{3} \phi_{z}=2 \pi c_{1}\left(E_{\rho}^{\prime}\right)
$$

where $\phi_{x}, \phi_{y}$, and $\phi_{z}$ are topological phases appearing in the quaternionic space of the eigenvectors of the ferromagnetic 3D Ising model [3,4,7]. Thus, for the 3D Ising models at finite temperature (with the nontrivial topological structure in the partition function), we have $\mathrm{n}_{1}=1, \mathrm{n}_{2}=\mathrm{n}_{3}=2, \phi_{x}=2 \pi, \phi_{y}=\phi_{z}=\pi / 2$, while $c_{1}\left(E_{\rho}^{\prime}\right)=2$. Note that the topological phases obtained above are catalogued to be the holonomy $\mathrm{k} \pi / 2$ ( $\mathrm{k}$ is an integer) of a connection, after parallel transport of a vector along a closed loop $\gamma$ on a Riemann manifold [41-51]. We have succeeded in fixing the topological phases appearing on the quaternionic space of the eigenvectors of the ferromagnetic 3D Ising model based on the Gauss-Bonnet-Chern formula with knowledge of the algebraic topology and differential geometry. This agrees with the phases of the local transformation as well as the gauge transformation, obtained in [7].

We can prove the following theorem:

Theorem 4. The 3D Ising model in the zero external magnetic field can be renormalized by use of the derivation of Gauu-Bonnet-Chern formula as a trivialization model on a nontrivial topological manifold.

The proof of Theorem 4 is represented in Appendix B.

As revealed above (and also in $[3,4,7,10,62-65]$, in three dimensions the polygon picture breaks down and one has to talk about knots. The nontrivial topological effect indeed exists in the 3D Ising model, and the global problems arise in the language of $\Gamma$ matrices. Although the Ising model with only the nearest-neighboring interactions looks like local in the original spin variable language, one cannot neglect the existence of the topological global effect in the system $[3,4,7,10]$. The situation is very similar to the Aharonov-Bohm (A-B) effect in which in the language of the magnetic field, no field, but in the language of potential, the A-B effect exists [66]. However, one cannot say that the A-B effect does not exist in the system (even in the language of the magnetic field). The A-B effect revealed in the language of potential is an observable quantity of topological phases with physical significance. Similarly, the nontrivial topological effect revealed in the language of $\Gamma$ matrices for the 3D Ising model is also an observable quantity with physical significance $[3,4,7,10]$. One cannot neglect its existence even in the original spin variable language. In other words, we think that the original spin variable language is not a good representation for studying the 3D Ising models, as is the language of the magnetic field for the A-B effect. It is our opinion that to further inspect the physical significance of the topological phases generalized on the eigenvectors of the 3D Ising model, it is reasonable to mention the similarity between the topological/geometrical phases in the 3D Ising model and Chern-Simons, Yang-Mills, Aharonov-Bohm, Berry phases, etc., as did in $[4,7,67]$.

Summarizing the discussions of this paper, we have proven the following Theorem:

Theorem 5. (Main Theorem). The Zhang's conjecture 2 can be solved. Let $M$ be a manifold, $p_{0} \in M$ and let $\pi_{1}\left(M, p_{0}\right)$ be the fundamental group of $M$ based at $p_{0}$. Consider a monodromy representation: $\rho: \pi_{1}\left(M, p_{0}\right) \rightarrow G L(M, C)$. Consider the representation for the ferromagnetic $3 D$ Ising model in the zero external magnetic field: 


$$
\widetilde{\rho}: \pi_{1}\left(R_{g}-\left\{a_{1}, \ldots, a_{N}\right\}\right) \otimes C l\left(I_{3 D}\right) \rightarrow C L^{*}\left(I_{3 D}\right)
$$

with singularities $a_{i}(I=1, \ldots, N)$. The vertex operators can be introduced to represented knots of types I and II for the 3D Ising model. Then a solution of the Riemann-Hilbert problem gives a flat connection. The model with can be renormalized by use of the derivation of the Gauss-Bonnet-Chern formula in cooperation with the monoidal transforms in a $4 D$ Riemann manifold. Three topological phases $\phi_{x}=2 \pi, \phi_{y}=\phi_{z}=\pi / 2$ appear on the quaternionic eigenvectors of the 3D Ising model on a nontrivial topological manifold in the $(3+1)$ dimensions.

\section{Interplay between the Physical Properties of Many-body Interacting Systems, Algebra, Topology, and Geometry}

In this section, we first summarize our results obtained in this work: a mapping between a knot and a spin lattice can be introduced to investigate the contribution of nontrivial topological structures to the partition function and physical properties of the ferromagnetic 3D Ising model, and the emergence of the additional dimension. This gives a concise proof of Zhang's conjectures 1 and 2, and uncovers the connection between different approaches, such as the Clifford algebra approach [7] and the Riemann-Hilbert problem method [10], developed based on algebra and topology. We then concentrate on a detailed proof of Zhang's conjecture 2 by applying the Riemann-Hilbert problem and Gauss-Bonnet-Chern formula. After describing the representation of the fundamental group, realizing the representation on the Riemann surface and formulating the RiemannHilbert problem, we introduce vertex operators of knot types and a flat vector bundle of the 3D Ising model. By applying the monoidal transforms, we trivialize the knots/links in a 4D Riemann manifold to obtain new trivial knots and proceed to obtain the renormalized model of the 3D Ising model. In order to eliminate these singularities from knots, we have to take off nontrivial knots in a higher dimensional space, by use of the derivation of GaussBonnet-Chern formula. We perform renormalization of the model and have the curvature structure in the model. The ferromagnetic 3D Ising model in the zero external magnetic field with nontrivial topological structures can be realized as the trivial model on a nontrivial topological manifold. The topological phases are determined by the Gauss-Bonnet-Chern formula, in consideration of the mathematical structure of the 3D Ising model, with regard to algebra, topology and geometry. Hence Zhang's conjecture 2 has been proven rigorously by applying the Riemann-Hilbert problem and the Gauss-Bonnet-Chern formula.

In what follows, we utilize the ferromagnetic 3D Ising model in the zero magnetic field as a platform to investigate the interplay between the physical properties of interacting many-body systems, algebra, topology and geometry.

Algebra: (1) The Clifford algebra representation for the transfer matrices of the 3D Ising model in the zero magnetic field reveals the nonlinear terms, which indicate the existence of the long-range entanglement between spins and the nontrivial topologic structure of the 3D Ising model $[3,4,7,10]$. (2) The K/C algebra can be defined to combine the knot and the Clifford algebra to represent the knot structure of the 3D Ising model [10]. (3) The Jordan algebra $[68,69]$ is employed to deal with non-commutative operators (such as $\Gamma$ matrices), to set up the system with the Jordan-von Neumann-Wigner framework [70], and to include the time average [7]. (4) The Riemann-Hilbert problem method is employed to solve the differential equation with singularities. (5) The quaternion algebra is needed to construct quaternionic eigenvectors so that the quaternionic topological/geometric phases emerge in quaternionic Hilbert space as a result of the topological transformation and the monodromy representation $[3,4,7]$. The quaternion basis naturally represents a rotation in $(3+1) \mathrm{D}$ space-time. (6) The Lee algebra represents the rotations in the 2D plane and also the rotations in subspaces of the $4 \mathrm{D}$ Riemann manifold $[3,4,7]$.

Topology: (1) The nontrivial topologic structure exists in the 3D Ising model without the external magnetic field, which represents the long-range entanglement between spins $[3,4,7,8,10,71,72]$, even if only the nearest-neighboring spin interactions are considered in the system. (2) The partition function of the 3D Ising model in the zero magnetic field consists of two kinds of components, i.e., spin alignment component and knot compo- 
nent $[3,4,7,10,13-19]$. (3) The "big" topological structure consists of an original topological structure and an "imagine" topological structure is trivialized naturally in the $(3+1) \mathrm{D}$ or 4D space. (4) The topological contribution to the free energy and other thermodynamic physical properties can be computed by a topological transformation as well as a gauge transformation [7], or a monoidal transformation [10], which is connected to the Jones polynomial $[17,73]$ with the formulas of Wilson loop [74,75] and Witten integral [76-79] for the action of the gauge group.

Geometry: (1) The 4D Riemann manifold is introduced to realize the knots on the Riemann surface, to formulate the representation of the Riemann-Hilbert problem, to apply the monoidal transformations at the knot intersection (singular) points, and to produce the trivialization of the knots [10]. (2) The geometrical structure of the 3D Ising model is described as geometric relations in hyperbolic 3-sphere (or 4-ball) represented in a $(3+1)$ D Poincaré ball model $[3,4,7]$, which is an extension of a hyperbolic triangle in the 2D Poincaré disk model for the 2D Ising system [2,11]. (3) The topological/geometric phase factors are generated on the eigenvectors of the 3D Ising model in the zero external magnetic field, which are analogous to the phase factors in the Aharonov-Bohm effect [66], the Berry phase effect [41,42] and fractional quantum Hall effect, etc. [42].

Dimensions: The ferromagnetic 3D Ising model in the zero external magnetic field can be solved in a $4 \mathrm{D}$ space. The additional dimension can be added in several approaches [3,4,7,10]: (1) the time average should be taken into account in addition to the ensemble average and quantum-mechanical average for a quantum statistic many-body interacting spin system, which is carried out with the Jordan-von Neumann-Wigner framework with Jordan algebra [7]. (2) The dimension is expanded by adding k terms of unit matrices in the terms of the direct product of original transfer matrices and adjusting the sequence of the unit matrices with other matrices, while keeping the trace invariance [7]. (3) The realizations of knots in the partition function are produced on a 4D Riemann manifold and by monoidal transform [10]. (4) By using a one-to-one mapping between a knot and a spin lattice, the original 3D Ising lattice and the "imagine" Ising lattice together construct a $(3+1) \mathrm{D}$ Ising lattice [4] (see also Section 2).

Topological transformations: (1) A topological transformation is applied by a matrix or a rotation to transform the nontrivial topological basis to the trivial topological basis, and vice versa $[3,4,7,10]$. (2) A local transformation, being a gauge transformation, is employed to trivialize the nontrivial knot structure [7]. (3) A monoidal transform is applied to trivialize the knots/links in a 4D Riemann manifold and obtain new trivial knots [10]. (4) Regarding the integrability of the system, the Reidemeister moves correspond to the Yang-Baxter equations for the topological transformation in the 2D Ising models, while the Reidemeister moves together with the disconnection and fusion of crossings correspond to the generalized Yang-Baxter equations (namely, the tetrahedron equations) for the topological transformation in the 3D Ising models [4].

Topological phases: (1) The topological phases are generalized on wavevectors as a result of the time average as a monodromy representation. (2) The topological phases are generalized by a local transformation on the gauge system. (3) The topological phases are generalized by renormalizing the ferromagnetic 3D Ising model by application of the Riemann-Hilbert problem method and use of the derivation of the Gauss-Bonnet-Chern formula. (4) The topological phases are generalized by the disconnection and fusion of crossings in the tetrahedron equations, in order to keep the integrability. (5) The topological phases are generated by parallel displacement in general curved spaces, while the 3D Ising model with singularities can be described by the connection on a nontrivial $\mathrm{Z}_{2}$ bundle, with analogy to electromagnetism with monopole [80].

In our previous work [7], Zhang's two conjectures proposed in [3] were proven rigorously by the Clifford algebraic approach. Zhang's two conjectures were also proven in [10] and this work by application of the Riemann-Hilbert problem. However, the explicit expression for the resulting partition function $\mathrm{Z}$ was not provided directly by the present procedure of the Riemann-Hilbert problem, the monoidal transformations and the Gauss- 
Bonnet-Chern formula. It should be emphasized that the results obtained by applying anyone of these approaches developed in $[7,10]$ and this work must be equivalent. Thus, the partition function obtained in $[3,4]$, which has been proven rigorously by the Clifford algebra approach [7], can be used to guard determination of the nontrivial knot components of the partition function in the monoidal transform trivialization. It is noticed that in the Clifford algebraic approach [7], we utilized the Largest Eigenvalue Principle that in the thermodynamic limit, only the largest eigenvalue contributes dominantly to the partition function of the 3D Ising model. With this constraint, we picked up the desired solution among all $2^{\text {nl }}$ possible solutions in $2^{\text {nl }}$ sub-spaces produced by the direct product of all the sub-transfer matrices. The combination of the local transformation and the Largest Eigenvalue Principle solves the problems of overdetermined tetrahedron equations. In the present procedure of the Riemann-Hilbert problem, we may also need to employ the Largest Eigenvalue Principle to make a constraint on possible solutions, since the nontrivial topological structure is very complicated and the monoidal transforms involved are indeed too many, causing a great number of possible solutions. It will be of great interest to derive a rigorous formulation for the nontrivial knots' components of the partition function $\mathrm{Z}$ by the procedure of the Riemann-Hilbert problem. Clearly, the 3D Ising model will be a good platform for an interplay between the physical properties of interacting many-body systems, algebra, topology, and geometry.

Remark 8. Wu and Yang [80] formulated the Aharonov-Bohm effect [66] in terms of a nonintegrable phase factor for electromagnetism in a space-time region, regarding Dirac' s magnetic monopole field. It was generalized to the gauge field theory with non-Abelian groups, which resulted in identification with the mathematical concept of connections on principal fiber bundles. Specially, the gauge potential is identification with a connection on a principal fiber bundle. In our present work, we established the relation between the Riemann-Hilbert problem of the 3D Ising model with singularities, the Gauss-Bonnet-Chern formula in a monodromy representation and the parallel displacement of a tangent vector in general curved spaces. It also sets up some correlations between the topological phases of the 3D Ising model, the connections on flat vector bundles and the curvature. In this way, the 3D Ising model is also associated with the gauge field theory, which can be mapped to a $3 D Z_{2}$ lattice gauge theory [81-84].

Remark 9. In this work and also [7], we have proven that the three topological phases appearing on the quaternionic eigenvectors of the $3 D$ Ising model are $\phi_{x}=2 \pi, \phi_{y}=\phi_{z}=\pi / 2$, respectively. It should be pointed out that, usually, inter-exchanging two particles (or spins) in three dimensions one obtains a phase factor of $2 \pi$ or $\pi$ for bosons or fermions, and only in $2 D$ a fractional statistics with a phase factor of other angles can be possible for anyons. The phase factor $\pi / 2$ for the $3 D$ Ising model is caused by inter-exchanging two spins in the quasi-2D limit, since the $3 D$ Ising model can be extended to be $(3+1) D$ and then separated to be many quasi-2D Ising models (see Theorem 1 in [7]). It is a global effect of many-body interactions between spins in a 3D lattice.

\section{Conclusions}

In conclusion, in the preceding paper [10] and this paper, we developed a method of the Riemann-Hilbert problem to rigorously prove Zhang's two conjectures proposed in [3] for the ferromagnetic 3D Ising model in the zero external magnetic field. In this work, we introduced vertex operators of the knot types and a flat vector bundle of the 3D Ising model. By applying the monoidal transforms to trivialize the knots/links in a $4 \mathrm{D}$ Riemann manifold and obtain new trivial knots [10], we renormalized the ferromagnetic 3D Ising model in the zero magnetic field by use of the derivation of the Gauss-BonnetChern formula. The 3D Ising model with nontrivial topological structures in the partition function can be realized as a trivial model on a nontrivial topological manifold. The results verify the correctness of Zhang's exact solution [3], which are consistent with the Clifford algebraic approach developed in [7]. Therefore, the exact solution of the ferromagnetic 3D Ising model in the zero external magnetic field is achieved in [3], in which one can find the explicit expressions for the partition function and the physical properties (such as 
the free energy, the spontaneous magnetization, the susceptibility, the specific heat, the spin correlation, the critical exponents). By inspecting/comparing the different procedures developed in $[7,10]$ and this paper, we showed that the 3D Ising model serves as a good platform for describing a sensible interplay between the physical properties of interacting many-body systems, algebra, topology, and geometry.

Author Contributions: Propose the project, Z.Z.; Physical ideas, Z.Z.; Mathematical methods, O.S. and Z.Z.; Proof of Theorem 1, Z.Z.; Proofs of Theorems 2 and 4, O.S. and Z.Z.; Proof of Theorem 3, Z.Z. and O.S.; Writing the paper, Z.Z. and O.S. All authors have read and agreed to the published version of the manuscript.

Funding: This research was funded by the National Natural Science Foundation of China under grant number 52031014. The APC was funded by the National Natural Science Foundation of China.

Institutional Review Board Statement: Not applicable.

Informed Consent Statement: Not applicable.

Data Availability Statement: The data is available on reasonable request from the corresponding author.

Conflicts of Interest: The authors declare no conflict of interest.

\section{Appendix A}

Proof of Theorem 2. Choosing an element $\Gamma$, we introduce the vertex operator [33-39]:

$$
V_{\alpha}^{(+)}(\Gamma, z)=\exp \left(\alpha \Gamma \sum_{n=1}^{\infty} \frac{a_{n}}{\sqrt{n}} z^{n}\right)
$$

where $\left\{a_{n}\right\}$ is the bosonic field:

$$
a_{n} a_{m}^{*}-a_{m}^{*} a_{n}=\delta_{n m} 1
$$

Other elements commute each other. By

$$
V_{\beta}^{(-)}\left(\Gamma^{\prime}, w\right)=\exp \left(\beta \Gamma^{\prime} \sum_{n=1}^{\infty} \frac{a_{n}^{*}}{\sqrt{n}} w^{-n}\right)
$$

we introduce the conjugate vertex operator. The product of the vertex operators is defined as follows:

$$
V_{\alpha}^{(+)}(\Gamma, z) \circ V_{\beta}^{(-)}(\bar{\Gamma}, w)=\exp (\alpha \Gamma) \circ \exp (\beta \bar{\Gamma}) \exp \left(\alpha \sum_{n=1}^{\infty} \frac{a_{n}}{\sqrt{n}} z^{n}\right) \exp \left(\beta^{\prime} \sum_{n=1}^{\infty} \frac{a_{n}^{*}}{\sqrt{n}} w^{-n}\right)
$$

Then the following equality can be proven:

$$
\left\langle 0\left|V_{\alpha}^{(+)}(\Gamma, z) \circ V_{\beta}^{(-)}(\bar{\Gamma}, w)\right| 0\right\rangle=\exp (\alpha \beta \Gamma \bar{\Gamma}) \cdot\left(1-\frac{z}{w}\right)^{\alpha \beta}
$$

By use of the vertex operators, we can describe the representation which we discussed in Section 3.

We give some basic properties of the vertex operators

(1) Putting $w=a_{j}, \alpha=i K . \beta=1, \Gamma=\Gamma_{j}, \bar{\Gamma}=\bar{\Gamma}_{j}$, we have

$$
\left\langle 0\left|V_{i K}^{(+)}\left(\Gamma_{j}, z\right) \circ V_{1}^{(-)}\left(\bar{\Gamma}_{j}, a_{j}\right)\right| 0\right\rangle=\exp \left(i K \Gamma_{j} \bar{\Gamma}_{j}\right) \cdot\left(a_{j}-z\right)^{i K} \cdot a_{j}^{-i K}
$$

Hence the product describes the monodromy representation near $a_{\mathrm{j}}$.

(2) We have 


$$
\left\langle 0\left|\prod_{k=1}^{N} V_{i K}^{(+)}\left(\Gamma_{k}, z\right) \circ V_{1}^{(-)}\left(\vec{\Gamma}_{k}, a_{k}\right)\right| 0\right\rangle=\exp \left(i K \Gamma_{1} \bar{\Gamma}_{1} \ldots \Gamma_{N} \bar{\Gamma}_{N}\right) \cdot\left(a_{1} \ldots a_{N}\right)^{-i K} \cdot \prod_{k=1}^{N}\left(a_{k}-z\right)^{i K}
$$

(3) The basic forms of knot types I and II can be described in terms of vertex operators:

i. $\quad$ The basic form of type I:

$$
\left\langle 0\left|\prod_{k=1}^{N} V_{i K}^{(+)}\left(\Gamma_{k}, z\right) \circ V_{1}^{(-)}\left(\vec{\Gamma}_{k}, a_{k}\right)\right| 0\right\rangle
$$

ii. The basic form of type II:

$$
\left\langle 0\left|V_{i K}^{(+)}\left(\Gamma_{1}, z\right) \prod_{k=2}^{N} V_{i K}^{(+)}\left(\Gamma_{k}, z\right) \circ V_{1}^{(-)}\left(\vec{\Gamma}_{k}, a_{k}\right) \times V_{1}^{(-)}\left(\bar{\Gamma}_{1}, a_{1}\right)\right| 0\right\rangle
$$

\section{Appendix B}

Proof of Theorem 4. We shall construct the renormalization model for the trivialization model. At first we are concerned with the renormalization of the basic forms.

(1) The basic form of type I.

The basic form of type I arises from the linear operator (matrix). Hence we are not concerned with type I.

(2) The basic form of type II.

The basic form of type II with a non-trivial topological structure can be trivialized and it is decomposed as follows: expiK $\left(\Gamma_{1} \Gamma_{2} \Gamma_{3} \ldots \ldots \Gamma_{\mathrm{n}}\right)$ and its conjugated term $\operatorname{expiK}\left(\bar{\Gamma}_{1} \bar{\Gamma}_{2} \overline{\bar{\Gamma}}_{3} \ldots \ldots \bar{\Gamma}_{\mathrm{n}}\right)$ can be reduced to the linear term $\operatorname{expiK}\left(\Gamma_{1} \Gamma_{2}\right)$ and $\operatorname{expiK}\left(\bar{\Gamma}_{1} \bar{\Gamma}_{2}\right)$ by use of the cancelation mechanism. At first, we analyze the divergence in $\left(\Gamma_{\mathrm{j}} \bar{\Gamma}_{j}\right)$ $(\mathrm{j}=3, \ldots \ldots, \mathrm{n})$. We analyze the divergence that arises from monoidal transform $[10,23,24,85-93]$ by use of the analytic realization (Figure A1):

Original realization

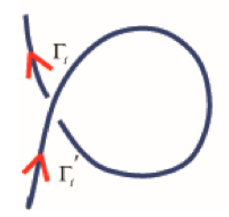

$\exp \left(i K \Gamma_{i} \Gamma_{i}^{\prime}\right)$

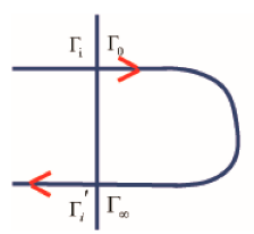

Analytic realization

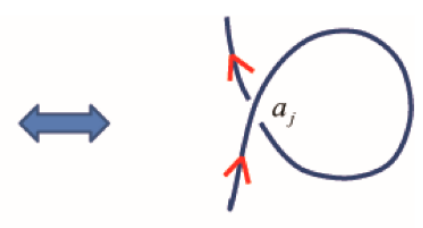

$$
\left(z-a_{i}\right)
$$

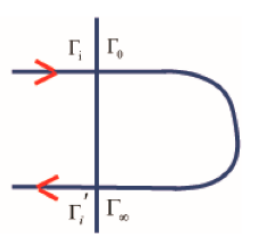

Figure A1. Divergence arises from the monoidal transform by use of the analytic realization.

Taking the correspondence into an account, we can analyze $\widetilde{W}$ at $\mathrm{z}=a_{j}$, by use of the monoidal transform $[10,23,24]$. Then we have:

$$
\mathrm{Q}^{*} \mathrm{w}^{\mathrm{A}}=\left\{\begin{array}{lll}
\mathrm{u}^{A} & \text { on } & V_{0} \\
\left(u^{\prime} v^{\prime}\right)^{A} & \text { on } & V_{\infty}
\end{array}\right.
$$


Hence $\Gamma_{\infty}=\Gamma^{\prime}, \Gamma_{0}=\Gamma^{\prime}$.

At first we notice the following behavior of $\widetilde{W}$ at $\mathrm{z}=a_{j}$ :

$$
\widetilde{W} \approx\left(z-a_{j}\right)^{\beta_{j}} \Pi \exp \left(i K \Gamma_{i} \Gamma_{i+1}\right)
$$

After we make the monoidal transform at $\mathrm{z}=a_{\mathrm{j}}$, we have

$$
Q^{*}(\widetilde{W})=\left\{\begin{array}{lll}
u_{j}^{\beta_{j}} \Pi \exp \left(i K \Gamma_{i} \Gamma_{i+1}\right) & \text { on } \hat{U}_{0} \\
u^{\prime}{ }_{j}^{\beta_{j}} v_{j}^{\prime}{ }_{j}{ }_{j} \Pi \exp \left(i K \Gamma_{i} \Gamma_{i+1}\right) & \text { on } \hat{U}_{\infty}
\end{array}\right.
$$

Here the cancellation mechanism is applied. Putting

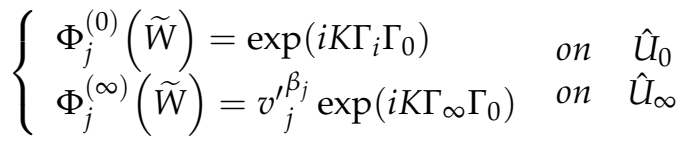

and making the vector bundle

$$
\widetilde{E}_{\rho_{0}}=\left\{\phi_{0 \infty}^{j}\right\}
$$

where

$$
\phi_{0 \infty}^{j}=\frac{\Phi_{j}^{(0)}(\widetilde{W})}{\Phi_{j}^{(\infty)}(\widetilde{W})}
$$

It is seen that $\left\{\Phi_{j}^{(0)}, \Phi_{j}^{(\infty)}\right\}$ constitute a section of $\widetilde{E}_{\rho}$, which has no more singularities. The anomaly can be calculated by applying the scheme in Section 5 . The calculus is complicated and omitted.

\section{References}

1. Ising, E. Report on the theory of ferromagnetism. Z. Phys. 1925, 31, 253-258. [CrossRef]

2. Onsager, L. Crystal Statistics I: A two-dimensional model with an order-disorder transition. Phys. Rev. 1944, 65, 117-149. [CrossRef]

3. Zhang, Z.D. Conjectures on the exact solution of three-dimensional (3D) simple orthorhombic Ising lattices. Philos. Mag. 2007, 87, 5309-5419. [CrossRef]

4. Zhang, Z.D. Mathematical structure of three-dimensional (3D) Ising model. Chin. Phys. B 2013, 22, 030513. [CrossRef]

5. Ławrynowicz, J.; Marchiafava, S.; Niemczynowicz, A. An approach to models of order-disorder and Ising lattices. Adv. Appl. Clifford Algebras 2010, 20, 733-743. [CrossRef]

6. Ławrynowicz, J.; Suzuki, O.; Niemczynowicz, A. On the ternary approach to Clifford structures and Ising lattices. Adv. Appl. Clifford Algebras 2012, 22, 757-769. [CrossRef]

7. Zhang, Z.D.; Suzuki, O.; March, N.H. Clifford algebra approach of 3D Ising model. Adv. Appl. Clifford Algebras 2019, $29,12$. [CrossRef]

8. Zhang, Z.D. Computational complexity of spin-glass three-dimensional (3D) Ising model. J. Mater. Sci. Tech. 2020, 44, 116-120. [CrossRef]

9. Zhang, Z.D. Exact solution of two-dimensional (2D) Ising model with a transverse field: A low-dimensional quantum spin system. Phys. E Low-Dimens. Syst. Nanostruct. 2021, 128, 114632. [CrossRef]

10. Suzuki, O.; Zhang, Z.D. A method of Riemann-Hilbert problem for Zhang's conjecture 1 in a ferromagnetic 3D Ising model: Trivialization of topological structure. Mathematics 2021, 9, 776. [CrossRef]

11. Kaufman, B. Crystal Statistics II: Partition function evaluated by spinor analysis. Phys. Rev. 1949, 76, 1232-1243. [CrossRef]

12. Lou, S.L.; Wu, S.H. Three-Dimensional Ising model and transfer matrices. Chin. J. Phys. 2000, 38, 841-854.

13. Kauffman, L.H. Knots and Physics, 3rd ed.; World Scientific Publishing Co. Pte. Ltd.: Singapore, 2001.

14. Kauffman, L.H. Knot Theory and Physics. In The Encyclopedia of Mathematical Physics; Francoise, J.P., Naber, G.L., Tsun, T.S., Eds.; Elsevier: Amsterdam, The Netherlands, 2007.

15. Kauffman, L.H. The mathematics and physics of knots. Rep. Prog. Phys. 2005, 68, 2829-2857. [CrossRef]

16. Lidar, D.A. On the quantum computational complexity of the Ising spin glass partition function and of knot invariants. New J. Phys. 2004, 6, 167. [CrossRef]

17. Jones, V.F.R. On knot invariants related to some statistical mechanical models. Pac. J. Math. 1989, 137, 311-336. [CrossRef]

18. Kauffman, L.H. State models and the Jones polynomial. Topology 1987, 26, 395-407. [CrossRef] 
19. Kauffman, L.H. New invariants in the theory of knots. Am. Math. Mon. 1988, 95, 195-242. [CrossRef]

20. Nechaev, S. Statistics of knots and entangled random walks. In Topological Aspects of Low Dimensional Systems; Springer: Berlin/Heidelberg, Germany, 2000; pp. 1-79.

21. Röhrl, H. Das Riemannsch-Hilbertsche Problem der Theorie der Linieren Differentialgleichungen. Math. Ann. 1957, 133, 1-25. [CrossRef]

22. Suzuki, O. The Problem of Riemann and Hilbert and the Relations of Fuchs in Several Complex Variables, Lecture Notes in Math; Springer: Berlin, Germany, 1977; Volume 712, pp. 325-364.

23. Lipman, J. Desingularization of two-dimensional schemes. Ann. Math. 1978, 107, 151-207. [CrossRef]

24. Hartshone, R. Algebraic Geometry, Graduate Texts in Math; Springer: New York, NY, USA, 1977; Volume 52.

25. Perk, J.H.H. Comment on "Mathematical structure of the three-dimensional (3D) Ising model". Chin. Phys. B 2013, 22, 131507. [CrossRef]

26. Francesco, P.D.; Mathieu, P.; Sénéchal, D. Conformal Field Theory; Springer: New York, NY, USA, 1997.

27. Polyakov, A.M. Conformal symmetry of critical fluctuations. JETP Lett. 1970, 12, 381.

28. Belavin, A.A.; Polyakov, A.M.; Zamolodchikov, A.B. Infinite conformal symmetry in two-dimensional quantum-field theory. Nucl. Phys. B 1984, 241, 333-380. [CrossRef]

29. Gaberdiel, M.R. An introduction to conformal field theory. Rep. Prog. Phys. 2000, 63, 607-667. [CrossRef]

30. Ginsparg, P. Applied Conformal Field Theory. In Fields, Strings and Critical Phenomena; Les Houches, Session XLIX, 1988; Brézin, E., Zinn-Justin, J., Eds.; Elsevier: Amsterdam, Netherlands, 1989.

31. Dijkgraaf, R.; Verlinde, E.; Verlinde, H. C = 1 conformal field theories on Riemann surfaces. Commun. Math. Phys. 1988, 115, 649-690. [CrossRef]

32. Moore, G.; Seiberg, N. Classical and quantum conformal field theory. Commun. Math. Phys. 1989, 123, 177-254. [CrossRef]

33. Tsuchiya, A.; Kanie, Y. Vertex operators in the conformal field theory on $\mathrm{P}^{1}$ and monodromy representations of the braid group. Lett. Math. Phys. 1987, 13, 303-312. [CrossRef]

34. Dimofte, T.; Gukov, S.; Hollands, L. Vortex counting and Lagrangian 3-manifolds. Lett. Math. Phys. 2011, 98, 225-287. [CrossRef]

35. Frenkel, I.B. Two constructions of affine Lie algebra representations and Boson-Fermion correspondence in quantum field theory. J. Funct. Anal. 1981, 44, 259-327. [CrossRef]

36. Li, H.S. Local systems of vertex operators, superalgebras and modules. J. Pure Appl. Algebra 1996, 109, 143-195. [CrossRef]

37. Davies, B.; Foda, O.; Jimbo, M.; Miwa, T.; Nakayashiki, A. Diagonalization of the XXZ Hamiltonian by vertex operators. Commun. Math. Phys. 1993, 151, 89-153. [CrossRef]

38. Frenkel, I.B.; Lepowsky, J.; Meurman, A. A natural representation of the Fischer-Griess Monster with the modular function J as character. Proc. Natl. Acad. Sci. USA 1984, 81, 3256-3260. [CrossRef] [PubMed]

39. Frenkel, I.B.; Jing, N. Vertex representations of quantum affine algebras. Proc. Natl. Acad. Sci. USA 1988, 85, 9373-9377. [CrossRef] [PubMed]

40. Zhang, Z.D.; March, N.H. Conformal invariance in the three dimensional (3D) Ising model and quaternionic geometric phase in quaternionic Hilbert space. arXiv 2011, arXiv:1110.5527.

41. Berry, M.V. Quantal phase factors accompanying adiabatic changes. Proc. R. Soc. London. A. Math. Phys. Sci. $1984,392,45-57$.

42. Barrett, T.W. Topological Foundations of Electromagnetism; World Scientific: Singapore, 2008.

43. Chen, L.; Rong, Y. Digital topological method for computing genus and the Betti numbers. Topol. Its Appl. 2010, 157, 1931-1936. [CrossRef]

44. Grinfeld, P. Introduction to Tensor Analysis and the Calculus of Moving Surfaces; Springer: Berlin/Heidelberg, Germany, 2014.

45. Ramadas, T.R. Chern-Simons gauge theory and projectively flat vector bundles on $\mathrm{M}_{\mathrm{g}}$. Commun. Math. Phys. 1990, 128, 421-426. [CrossRef]

46. Chern, S.S. On the curvatura integra in a Riemannian manifold. Ann. Math. 1945, 46, 674-684.

47. Borel, A.; Serre, J.P. Le théorème de Riemann-Roch. Bull. Société Mathématique Fr. 1958, 86, 97-136. [CrossRef]

48. Griffiths, P.; Harris, J. Principles of Algebraic Geometry; Wiley Classics Library; John Wiley and Sons: New York, NY, USA, 1994.

49. Berthelot, P.; Jussila, O.; Grothendieck, A.; Raynaud, M.; Kleiman, S.; Illusie, L. Théorie des Intersections et Théorème de Riemann-Roch; (SGA 6), LNM 225; Springer: Berlin, Germany, 1971.

50. Jürgen, J. Compact Riemann Surfaces; Springer: Berlin, Germany; New York, NY, USA, 2006; pp. $208-219$.

51. Porteous, I.R. Geometric Differentiation; Cambridge University Press: Cambridge, UK, 1994.

52. Fuchs, D.B.; Gabrielov, A.M.; Gel'fand, I.M. The Gauss-Bonnet theorem and the Atiyah-Patodi-Singer functionals for the characteristic classes of foliations. Topology 1976, 15, 165-188. [CrossRef]

53. Buzano, R.; Nguyen1, H.T. The higher-dimensional Chern-Gauss-Bonnet formula for singular conformally flat manifolds. J. Geom. Anal. 2019, 29, 1043-1074. [CrossRef]

54. Leuzinger, E. A Gauss-Bonnet formula for moduli spaces of Riemann surfaces. Geom. Dedicata 2016, 180, 373-383. [CrossRef]

55. Fuchs, L.I. Zur theorie der linearen differentialgleichungen mit veränderlichen coeffizienten. J. Rein. Angew. Math. 1866, 66, 121-160.

56. Saito, T. On Fuchs' relation for the differential equation with algebraic coefficients. Kodai Math. Sem. Rep. 1958, 10, 101-104. [CrossRef]

57. Corel, E. Exponents of a meromorphic connection on a compact surface. Pac. J. Math. 2009, 242, 259-279. [CrossRef] 
58. Albin, P. A renormalized index theorem for some complete asymptotically regular metrics: The Gauss-Bonnet theorem. Adv. Math. 2007, 213, 1-52. [CrossRef]

59. Mazzeo, R. Elliptic theory of differential edge operators I. Comm. Partial. Differ. Equ. 1991, 16, 1615-1664.

60. Alty, L.J. The generalized Gauss-Bonnet-Chern theorem. J. Math. Phys. 1995, 36, 3094-3105. [CrossRef]

61. Røgen, P. Gauss-Bonnet's theorem and closed Frenet frames. Geom. Dedicata 1998, 73, 295-315. [CrossRef]

62. Bick, E.; Steffen, E.D. Topology and Geometry in Physics; Springer: Berlin, Germany, 2005.

63. Bohm, A.; Mostafazadch, A.; Koizumi, H.; Niu, Q.; Zwanziger, J. The Geometric Phase in Quantum Systems; Foundations, Mathematical Concepts and Applications in in Molecular and Condensed Matter Physics; Springer: Berlin, Germany, 2003.

64. Newell, G.F.; Montroll, E.W. On the theory of the Ising model with ferromagnetism. Rev. Mod. Phys. 1953, 25, 353-389. [CrossRef]

65. Istrail, S. Universality of intractability for the partition function of the Ising model across non-planar lattices. In Proceedings of the 32nd ACM Symposium on the Theory of Computing, Portland, OR, USA, 21-23 May 2000; pp. 87-96.

66. Aharonov, Y.; Bohm, D. Significance of electromagnetic potentials in the quantum theory. Phys. Rev. 1959, 115, 485-491. [CrossRef]

67. Zhang, Z.D. Response to "Comment on a recent conjectured solution of the three-dimensional Ising model". Philos. Mag. 2008, 88, 3097-3101. [CrossRef]

68. Jordan, P. Über eine Klasse nichtassoziativer hyperkomplexer Algebren. Nachrichten von der Gesellschaft der Wissenschaften zu Göttingen Mathematisch-Physikalische Klasse 1932, 1932, 569-575.

69. Jordan, P. Über Verallgemeinerungsm “oglichkeiten des Formalismus der Quantenmechanik. Nachrichten von der Gesellschaft der Wissenschaften zu Göttingen Mathematisch-Physikalische Klasse 1933, 1933, 209-217.

70. Jordan, P.; von Neumann, J.; Wigner, E. On an algebraic generalization of the quantum mechanical formalism. Ann. Math. 1934, 35, 29-64. [CrossRef]

71. Zhang, Z.D. The nature of three dimensions: Non-local behavior in the three-dimensional (3D) Ising model. J. Phys. Conf. Ser. 2017, 827, 012001. [CrossRef]

72. Zhang, Z.D. Topological effects and critical phenomena in the three-dimensional (3D) Ising model, Chapter 27. In Many-Body Approaches at Different Scales: A Tribute to Norman H. March on the Occasion of his 90th Birthday; Angilella, G.G.N., Amovilli, C., Eds.; Springer: New York, NY, USA, 2018.

73. Jones, V.F.R. The Jones Polynomial. In The Encyclopedia of Mathematical Physics; Francoise, J.P., Naber, G.L., Tsun, T.S., Eds.; Elsevier: Amsterdam, The Netherlands, 2007.

74. Wilson, K.G. Confinement of qnarks. Phys. Rev. D 1974, 10, 2445-2459. [CrossRef]

75. Makeenko, Y.M. Brief Introduction to Wilson Loops and Large N. Phys. At. Nucl. 2010, 73, 878-894. [CrossRef]

76. Witten, E. Topological sigma models. Commun. Math. Phys. 1988, 118, 411-449. [CrossRef]

77. Witten, E. Gauge-theories and integrable lattice models. Nucl. Phys. B 1989, 322, 629-697. [CrossRef]

78. Witten, E. Quantum-field theory and the Jones polynomial. Commun. Math. Phys. 1989, 121, 351-399. [CrossRef]

79. Witten, E. Gauge-theories, vertex models, and quantum groups. Nucl. Phys. B 1990, 330, 285-346. [CrossRef]

80. Wu, T.T.; Yang, C.N. Concept of nonintegrable phase factors and global formulation of gauge fields. Phys. Rev. D 1975, 12, 3845-3857. [CrossRef]

81. Wegner, F. Duality in generalized Ising models and phase transitions without local order parameters. J. Math. Phys. 1971, 12, 2259-2272. [CrossRef]

82. Kogut, J.B. An introduction to lattice gauge theory and spin systems. Rev. Mod. Phys. 1979, 51, 659-713. [CrossRef]

83. Savit, R. Duality in field theory and statistical systems. Rev. Mod. Phys. 1980, 52, 453-487. [CrossRef]

84. Zhang, Z.D. Exact solution of three-dimensional (3D) $Z_{2}$ lattice gauge theory. Eur. J. Phys. 2021, in press.

85. Kock, A. Strong functors and monoidal monads. Arch. Math. 1972, 23, 113-120. [CrossRef]

86. Baez, J.C. Higher dimensional algebraI. braided monoidal 2-categories. Adv. Math. 1996, 121, 196-244. [CrossRef]

87. Bespalov, Y.N. Crossed modules and quantum groups in braided categories. Appl. Categ. Struct. 1997, 5, 155-204. [CrossRef]

88. Bespalov, Y.; Drabant, B. Hopf (bi-)modules and crossed modules in braided monoidal categories. J. Pure Appl. Algebra 1998, 123, 105-129. [CrossRef]

89. Kapranov, M.; Voevodsky, V. Braided monoidal 2-categories and Manin-Schechtman higher braid groups. J. Pure Appl. Algebra 1994, 92, 241-267. [CrossRef]

90. Balteanu, C.; Fiedorowicz, Z.; Schwänzl, R.; Vogt, R. Iterated monoidal categories. Adv. Math. 2003, 176, 277-349. [CrossRef]

91. Joyal, A.; Street, R.; Verity, D. Traced monoidal categories. In Mathematical Proceedings of the Cambridge Philosophical Society; Cambridge University Press: Cambridge, UK, 1996; Volume 119, pp. 447-468.

92. Bichon, J.; Rijdt, A.D.; Vaes, S. Ergodic coactions with large multiplicity and monoidal equivalence of quantum groups. Commun. Math. Phys. 2006, 262, 703-728. [CrossRef]

93. Yetter, D.N. Quantum groups and representations of monoidal categories. In Mathematical Proceedings of the Cambridge Philosophical Society; Cambridge University Press: Cambridge, UK, 1990; Volume 108, pp. 261-290. 